\title{
Scaling Limits for Interacting Diffusions ${ }^{\star}$
}

\author{
S. R. S. Varadhan \\ Courant Institute of Mathematical Sciences, New York University, 251 Mercer Street, \\ New York, NY 10012, USA
}

Received February 26, 1990; in revised form June 22, 1990

\begin{abstract}
We consider a large number of particles diffusing on a circle interacting through a drift resulting from the gradient of a pair potential whose support is of the order of the interparticle distance. We derive a nonlinear bulk diffusion equation for the density of the particle distribution on the circle. The diffusion coefficient is determined as a function of density in terms of standard thermodynamical objects.
\end{abstract}

\section{Introduction}

In this article we study the hydrodynamic limit for interacting Brownian motions on the one-dimensional circle. The interaction is between pairs of particles and is repulsive in nature. The scaling is such that the range of the interaction is of the same order as interparticle distance and therefore each particle interacts with only a finite number of nearby particles at any given time.

We obtain a nonlinear bulk diffusion equation and the diffusion coefficient is naturally expressed in terms of the thermodynamic functions of our one dimensional system. The main limitations are the finiteness of volume that is forced because our basic space is the circle, the repulsive nature of the interactions that is assumed and that we are in one space dimension.

In this context the fluctuations around equilibrium have been studied earlier by $\mathrm{H}$. Spohn [5] and the self diffusion in equilibrium by Guo and Papanicolaou [1]. Their results have been derived for infinite volume and arbitrary space dimension. Of course these results deal essentially with equilibrium problems where a lot of control through estimates is available.

Our method is similar to the one used in Guo, Papanicolaou and Varadhan [2] and uses estimates based on entropy and its rate of change. Section 2 describes

* This research was supported by a grant from the National Science Foundation DMS-8901682 
the results precisely and Sects. 3-9 contain detailed proofs. Section 10 contains some results concerning the Gibbs measures of one dimensional systems. These results are all well known to the experts although it is difficult to find precise references to the results in the form we need. We have therefore included a quick exposition with sketches of proofs.

Along with S. Olla in [3] we have modified the methods of this article to extend these results to the case of interacting Ornstein-Uhlenbeck processes and they will appear in the next article.

\section{Summary}

We denote by $S$ the circle of unit circumference and consider a system of $N$ interacting Brownian motions with $S$ as a state space satisfying the following system of stochastic differential equations:

$$
d x_{i}(t)=-N \sum_{j: j \neq i} V^{\prime}\left(N\left(x_{i}(t)-x_{j}(t)\right) d t+d \beta_{i}(t), \quad i=1,2, \ldots, N .\right.
$$

Here, $\beta_{1}, \ldots, \beta_{N}$ are $N$ independent Brownian motions and $V(\cdot)$ is an even function on $R$ satisfying the following assumptions:

(i) $V \geqq 0, V(0)>0$ and $V$ has compact support.

(ii) $V$ is once continuously differentiable.

(iii) $V(\cdot)$ is repulsive in the sense that

$$
\psi(z)=-z V^{\prime}(z) \geqq 0 .
$$

Then the process $\left[x_{1}(t), \ldots, x_{N}(t)\right]$ is a Markov process of diffusion type on $S^{N}$, the $N$-fold copy of $S$, with an infinitesimal generator given by

$$
L_{N}=\frac{1}{2} \sum_{i=1}^{N} \frac{\partial^{2}}{\partial x_{i}^{2}}-N \sum_{j \neq i} V^{\prime}\left(N\left(x_{i}-x_{j}\right)\right) \frac{\partial}{\partial x_{j}} .
$$

It is easily verified that

$$
L_{N}=\frac{1}{2} \sum_{i=1}^{N}\left(e^{\sum_{j, k} V\left(N\left(x_{j}-x_{k}\right)\right)} \frac{\partial}{\partial x_{i}} e^{-\sum_{\jmath, k} V\left(N\left(x_{j}-x_{k}\right)\right)} \frac{\partial}{\partial x_{i}}\right),
$$

so that $L_{N}$ is formally symmetric with respect to the measure

$$
d \mu_{N}(x)=\frac{1}{Z_{N}} \exp \left[-\sum_{i, j} V\left(N\left(x_{i}-x_{j}\right)\right)\right] d x_{1} \ldots d x_{N}
$$

It is straightforward to verify that the Markov process $x(t)$ with $L_{N}$ as generator is in fact reversible with respect to the invariant measure $\mu_{N}$ given by (2.5). The constant $Z_{N}$ is for normalization and is so defined to make $\mu_{N}$ into a probability measure.

We start $x(0)$ with an initial distribution with density $f_{N}^{0}\left(x_{1}, \ldots, x_{N}\right)$ with respect to the invariant density $\mu_{N}$. The density at time $t$ with respect to $\mu_{N}$ is denoted by $f_{N}^{t}\left(x_{1}, \ldots, x_{N}\right)$ and is given as a solution of the forward equation

$$
\frac{\partial f_{N}^{t}}{d t}=L_{N} f_{N}^{t} \quad \text { with }\left.\quad f_{N}^{t}\right|_{t=0}=f_{N}^{0}
$$


The empirical distribution of the process at time $t$ is defined by

$$
\xi_{N}(t, A)=\frac{1}{N} \sum_{i=1}^{N} \chi_{A}\left(x_{i}(t)\right) \text { for } A \subset S,
$$

and $\xi_{N}(t)$ is viewed as a random measure on $S$. If we denote by $M_{1}(S)$ the space of probability measures on $S$, one can view $\xi_{N}(\cdot)$ as a stochastic process with values in $M_{1}(S)$. In view of the continuity of the trajectories, we can pick a time $T<\infty$ and consider the space $C\left[[0, T], M_{1}(S)\right]$ of measure valued continuous functions on $[0, T]$, and our basic markov process with initial density $f_{N}^{0}$ will induce a measure $Q_{N}$ on $C\left[[0, T], M_{1}(S)\right]$. Our main result is to show that under suitable assumptions on $f_{N}^{0}$, the measures $Q_{N}$ as $N \rightarrow \infty$ will concentrate on a single measure valued trajectory which is the solution of a certain nonlinear diffusion equation.

In order to describe this nonlinear diffusion equation we have to introduce some thermodynamic functions of one dimensional systems with pair interaction given by $V(\cdot)$. The partition function in a finite region $[0, l]$ with activity $\lambda$ is given by

$$
\hat{Z}(l, \lambda)=\sum_{n=0}^{\infty} \frac{e^{n \lambda}}{n !} \int_{0}^{l} \ldots \int_{0}^{l} e^{-\sum_{t,} V\left(x_{i}-x_{J}\right)} d x_{1} \ldots d x_{n} .
$$

It is known [4] that the free energy defined by

$$
F(\lambda)=\lim _{l \rightarrow \infty} \frac{\log \hat{Z}(l, \lambda)}{l}
$$

exists and is a convex function of $\lambda$ for all $\lambda$,

$$
\rho(\lambda)=\frac{d F}{d \lambda},
$$

is the "density" corresponding to the activity $\lambda$ and is a continuous strictly monotone function of $\lambda$. This function of course can be inverted to yield $\lambda=\lambda(\rho)$ as a function of $\rho$. The free energy expressed as a function of $\rho$, i.e.

$$
P(\rho)=F(\lambda(\rho))
$$

is called the pressure and is again a continuous strictly monotone function of the density $\rho$.

Our main result can now be stated. Let us assume that the initial densities $\left\{f_{N}^{0}\right\}$ have the following behavior as $N \rightarrow \infty$ :

$$
\begin{aligned}
& \int f_{N}^{0} \log f_{N}^{0} d \mu_{N} \leqq C N \text { for some } C<\infty \text { and all } N \text {; } \\
& \int_{A_{N, J, \varepsilon}} f_{N}^{0} d \mu_{N} \rightarrow 0 \text { as } N \rightarrow \infty
\end{aligned}
$$

for every smooth test function $J$ on $S$. Here $A_{N, J, \varepsilon}$ is the set of configurations $\left(x_{1}, \ldots, x_{N}\right)$ such that

$$
A_{N, J, \varepsilon}=\left\{x:\left|\frac{1}{N} \sum J\left(x_{i}\right)-\int J(\theta) \rho_{0}(\theta) d \theta\right|>\varepsilon\right\},
$$

and $\rho_{0}(\theta)$ is some fixed density on $S$ such that $\rho_{0}(\theta) \geqq 0$ and $\int \rho_{0}(\theta) d \theta=1$. In 
order for (2.12) and (2.13) to be compatible $\rho_{0}(\theta)$ will have to satisfy some minimal regularity conditions. In any case (2.13) asserts that

$$
\frac{\delta_{x_{1}}+\ldots+\delta_{x_{N}}}{N} \Rightarrow \rho_{0}(\theta) d \theta
$$

in the sense of weak convergence, in probability with respect to the initial distribution $f_{N}^{0} d \mu_{N}$.

Under these assumptions we establish that $Q_{N}$ converges weakly to a limit which is the degenerate distribution on a single trajectory in $M_{1}(S)$ described by $\rho(\theta, t) d \theta$, where $\rho(\theta, t)$ is defined as the unique solution (satisfying mild regularity conditions) of

with

$$
\frac{\partial \rho(\theta, t)}{\partial t}=\frac{1}{2}\left[P(\rho(\theta, t)]_{\theta \theta}\right.
$$

$$
\left.\rho(\theta, t)\right|_{t=0}=\rho_{0}(\theta)
$$

\section{Outline of Proof}

The proof of our result is based on the following ideas. For the sequence $\left\{Q_{N}\right\}$ of probability measures on the space $\Omega_{T}=C\left[[0, T], M_{1}(S)\right]$ of measure valued trajectories we first establish compactness under weak convergence.

Theorem 3.1. The sequence $\left\{Q_{N}\right\}$ is tight on the space $\Omega_{T}$.

In order to establish that the limit $Q_{N}$ exists and is concentrated on a single trajectory we establish properties satisfied by the support of any limit point $Q$, enough properties so that one is left with a single trajectory satisfying all of them.

Let us consider a smooth function $u(\theta)$ on $S$ and the corresponding functional

$$
\xi_{N}(t)=\frac{1}{N} \sum u\left(x_{i}(t)\right)
$$

By Itô's formula we see that

$$
\begin{aligned}
d \xi_{N}(t)= & \frac{1}{2 N} \sum_{i=1}^{N} u^{\prime \prime}\left(x_{i}(t)\right) d t \\
& -\sum_{i, j=1}^{N} V^{\prime}\left(N\left(x_{i}(t)\right)-x_{j}(t)\right) u^{\prime}\left(x_{i}(t)\right) d t \\
& +\frac{1}{N} \sum_{i=1}^{N} u^{\prime}\left(x_{i}(t)\right) d \beta_{i}(t)
\end{aligned}
$$

By the law of large numbers, as $N \rightarrow \infty$, the third term goes to zero. As for the second term 


$$
\begin{aligned}
& \sum_{i, j=1}^{N} V^{\prime}\left(N\left(x_{i}(t)-x_{j}(t)\right)\right) u^{\prime}\left(x_{i}(t)\right) \\
& \quad=\frac{1}{2} \sum V^{\prime}\left(N\left(x_{i}(t)-x_{j}(t)\right)\right)\left(u^{\prime}\left(x_{i}(t)\right)-u^{\prime}\left(x_{j}(t)\right)\right)
\end{aligned}
$$

(by skew symmetry of $V^{\prime}$ )

$$
\simeq \frac{1}{2} \sum V^{\prime}\left(N\left(x_{l}(t)-x_{j}(t)\right)\right) u^{\prime \prime}\left(x_{i}(t)\right)\left(x_{i}(t)-x_{j}(t)\right)
$$

by (mean value theorem)

$$
\simeq-\frac{1}{2 N} \sum \psi\left(N\left(x_{i}(t)-x_{j}(t)\right)\right) u^{\prime}\left(x_{i}(t)\right) \text {. }
$$

If we are at some point $(\theta, t)$ in space time and the local density of particles is $\rho(\theta, t)$ then the system should be in local equilibrium there in a Gibbs state with density $\rho$ and it is not a difficult calculation to show that in an exact Gibbs state of density $\rho$

$$
\lim _{l \rightarrow \infty} \frac{1}{l} \sum_{x_{l}, x_{\jmath} \in[0, l]} \psi\left(x_{i}-x_{\jmath}\right)=P(\rho)-\rho .
$$

One therefore hopes to estabish that if at a typical time $t, \frac{1}{N}\left[\delta_{x_{1}(t)}+\ldots+\delta_{x_{N}(t)}\right]$ is close to $\rho(t, \theta) d \theta$, then $\frac{1}{2 N} \sum \psi\left(N\left(x_{i}(t)-x_{j}(t)\right) u^{\prime \prime}\left(x_{i}(t)\right)+\frac{1}{2 N} \sum u^{\prime \prime}\left(x_{i}(t)\right)\right.$ should be close to $\frac{1}{2} \int P(\rho(t, \theta)) u^{\prime \prime}(\theta) d \theta$.

In this manner we establish

Theorem 3.2. Any limit point $Q$ of $Q_{N}$ satisfies

(i) $Q[\alpha(t, \cdot): \alpha(t, d \theta)=\rho(t, \theta) d \theta$ for a.e. $t]=1$.

(ii) $E^{Q}\left[\rho: \rho(0, \theta)=\rho_{0}(\theta)\right.$ a.e. $\left.\theta\right]=1$.

(iii) $Q\left[P: \int u(\theta) \rho(t, \theta) d \theta-\int u(\theta) \rho_{0}(\theta) d \theta=\frac{1}{2} \int_{0}^{t} \int u^{\prime \prime}(\theta) P(\rho(t, \theta)) d \theta\right]=1$.

Given Theorem 3.2, $Q$ lives on the set of weak solutions of

$$
\left\{\begin{array}{l}
\frac{\partial \rho}{\partial t}=\frac{1}{2}(P(\rho))_{\theta \theta} \text { with } \\
\left.\rho(t, \theta)\right|_{t=0}=\rho_{0}(\theta) .
\end{array}\right.
$$

One can establish the uniqueness of weak solutions of (3.3) under the assumptions

$$
\int_{0}^{T} \int_{S} \rho^{3}(t, \theta) d t d \theta<\infty
$$


and

$$
\int_{0}^{T} \int_{s} \frac{1}{\rho(t, \theta)}\left[\frac{\partial}{\partial \theta} P(\rho(t, \theta))\right]^{2} d \theta<\infty
$$

In order then to complete the proof we only need to establish

Theorem 3.3. Any limit point $Q$ satisfies

and

$$
E^{Q}\left[\int_{0}^{T} \int_{S} \rho^{3}(t, \theta) d t d \theta\right]<\infty
$$

$$
E^{Q}\left[\int_{0}^{T} \int_{S} \frac{1}{\rho(t, \theta)}\left[\frac{d}{d \theta} P(\rho(t, \theta))\right]^{2} d t d \theta\right]<\infty
$$

Finally a word about the strategy to be used in establishing Theorems 3.1., 3.2 and 3.3. Since $N \rightarrow \infty$ we need to develop estimates that hold uniformly in $N$. We will need properties of the solutions $f_{N}^{t}$, and $\tilde{f}_{N}=\frac{1}{T} \int_{0}^{T} f_{N}^{s} d s$, where
$f_{N}^{t}$ solves

$$
\frac{\partial f_{N}^{t}}{\partial t}=L_{N} f_{N}^{t}
$$

Our assumptions is that $\int f_{N}^{0} \log f_{N}^{0} d \mu_{N} \leqq C N$. One knows that

$$
\int f_{N}^{t} \log f_{N}^{t} d \mu_{N}=H_{N}(t)
$$

is nonincreasing in $t$ and

$$
\frac{d}{d t} H_{N}(t)=-I_{N}(t)=-\frac{1}{2} \int \frac{\left|\nabla f_{N}^{t}\right|^{2}}{f_{N}^{t}} d \mu_{N}
$$

The functionals

and

$$
H_{N}(t)=\int f \log f d \mu_{N}
$$

$$
I_{N}(t)=\frac{1}{2} \int \frac{|\nabla f|^{2}}{f} d \mu_{N}
$$

are lower semicontinuous, non-negative and convex. Therefore one gets easily

$$
\begin{aligned}
H_{N}\left(\bar{f}_{N}\right) & =H_{N}\left(\frac{1}{T} \int_{0}^{T} f_{N}^{s} d s\right) \\
& \leqq \frac{1}{T} \int_{0}^{T} H\left(f_{N}^{s}\right) d s \\
& \leqq H\left(f_{N}^{0}\right) \leqq C N
\end{aligned}
$$

Similarly, 


$$
\begin{aligned}
I_{N}\left(\bar{f}_{N}\right) & \leqq \frac{1}{T} \int_{0}^{T} I_{N}\left(f_{N}^{s}\right) d s \\
& =\frac{1}{T}\left[H_{N}\left(f_{N}^{0}\right)-H_{N}\left(f_{N}^{T}\right)\right] \\
& \leqq \frac{1}{T} H_{N}\left(f_{N}^{0}\right) \\
& \leqq \frac{C}{T} N .
\end{aligned}
$$

In some sense our procedures are based solely on the two estimates derived above.

\section{Some Estimates Based on Entropy}

Given a density $f_{N}$ relative to the invariant measure $\mu_{N}$ with entropy $H_{N}\left(f_{N}\right)=\int f_{N} \log f_{N} d \mu_{N}$, we shall obtain in this section some preliminary estimates based on a bound of the type

$$
H_{N}\left(f_{N}\right) \leqq A N
$$

Lemma 4.1. There exists a constant $C$ such that for every $N$ and every $f_{N}$ satisfying (4.1) we have

$$
E^{f_{N}}\left\{\frac{1}{N} \sum_{i, j} V\left(N\left(x_{i}-x_{j}\right)\right)\right\} \leqq A+C,
$$

where $A$ is the same constant as in (4.1).

Proof.

$$
\begin{aligned}
& E^{f_{N}}\left\{\frac{1}{N} \sum_{i_{j}} V\left(N\left(x_{i}-x_{j}\right)\right)\right\} \\
& \quad \leqq \frac{1}{N} \log E^{\mu_{N}}\left\{\exp \left[\sum V\left(N\left(x_{i}-x_{j}\right)\right)\right]\right\}+A \\
& \quad \leqq-\frac{1}{N} \log Z_{N}+A=-\frac{1}{N} \log \int e^{-\sum V\left(N\left(x_{i}-x_{j}\right)\right)} d x_{1} \ldots d x_{N}+A \\
& \quad \leqq \frac{1}{N} \int\left(\sum V\left(N\left(x_{i}-x_{j}\right)\right)\right) d x_{1} \ldots d x_{N}+A \\
& \quad \leqq C+A .
\end{aligned}
$$

From now on for the rest of the section we want to derive some consequences of an estimate of the form

$$
E^{f_{N}}\left\{\frac{1}{N} \sum V\left(N\left(x_{i}-x_{j}\right)\right)\right\} \leqq B \text { for all } N .
$$


Let us divide the circle $S$ into a large number $l$ of small arcs length $\frac{1}{l}$. Let us denote them by $S_{1}, S_{2}, \ldots, S_{l}$. If we are given a configuration of $N$ points $x_{1}, \ldots, x_{N}$ we can form the frequency counts

$$
g_{i}=\sum_{j=1}^{N} \chi_{s_{l}}\left(x_{j}\right)
$$

of the number of points among $x_{1}, \ldots, x_{N}$ that fall in a given arc $S_{i}$ for $1 \leqq i \leqq l$. We wish to estimate $\sum_{l=1}^{l} g_{i}^{2}$ in terms of $\sum_{i, j} V\left(N\left(x_{i}-x_{J}\right)\right)$. Let us subdivide each arc $S_{i}$ into $2 k$ arcs of length $\frac{1}{2 l k}$ and we assume that the size $\frac{1}{2 l k}$ is of the order $\frac{\delta}{N}$, where $\delta$ is small, independent of $N$, and is so small that $V(x) \geqq \eta$ for $|x| \leqq \delta$ for some positive constant $\eta>0$. For $1 \leqq i \leqq 2 l k$, if we denote by $h_{i}$ the frequency counts for the smaller arcs, then

$$
\sum_{1}^{l} g_{i}^{2} \leqq 2 k \sum_{1}^{2 l k} h^{2} i
$$

If we translate the basic arcs $S_{l}$ by half their lengths and denote them by $S_{i+1 / 2}$ with their frequency counts $g_{i+1 / 2}$, then we have just as well

$$
\sum_{1}^{l} g_{i+1 / 2}^{2} \leqq 2 k \sum_{1}^{2 l k} h_{i}^{2}
$$

If from among $x_{1}, \ldots, x_{N}$, we have a pair $x_{r}, x_{s}$ with $\left|x_{r}-x_{s}\right|$ $\leqq \frac{1}{2 l}$, then they must belong either to the same $S_{\imath}$ or $S_{i+1 / 2}$ for some $i$. Therefore

$$
\begin{aligned}
\sum_{i, j} \chi_{|x| \leqq 1 / 2}\left(\left|\left(x_{i}-x_{j}\right)\right|\right) & \leqq \sum g_{i}^{2}+\sum g_{i+1 / 2}^{2} \\
& \leqq(4 k) \sum_{1}^{2 l k} h_{i}^{2} \\
& \leqq \frac{4 k}{\eta} \sum V\left(N\left(x_{i}-x_{j}\right)\right) .
\end{aligned}
$$

If $W(x)$ is a function that is supported on $\left[-\frac{1}{2 l}, \frac{1}{2 l}\right]$ and is bounded there by a constant $\|W\|$ then

$$
\sum_{i, j} W\left(x_{i}-x_{J}\right) \leqq\|W\| \cdot \frac{4 k}{\eta} \sum V\left(N\left(x_{i}-x_{j}\right)\right)
$$

provided only that $\frac{N}{2 l k}=\delta$ is sufficiently small.

Lemma 4.2. If $W$ is a fixed compactly supported function then we can estimate

$$
E^{f_{N}} \frac{\lambda}{N^{2}} \sum W\left(\lambda\left(x_{i}-x_{j}\right)\right) \leqq\|W\| \cdot B \cdot C
$$


where $\|W\|$ is a bound for $W, B$ is any constant satisfying (4.2) and $C$ is a universal constant depending only on $V$ and the size of the support of $W(\cdot)$. This estimate holds uniformly for all $\lambda$ in the range $\varepsilon_{0} \leqq \lambda \leqq N$.

Proof. We need basically $l$ to be $c \lambda$ for fixed constant $c$ in (4.4) and that forces on us a choice of $k=C N / \lambda$ for some other constant $C$. Now Lemma 4.2 is an immediate consequence of (4.2) and (4.4).

\section{Some Estimates Based on the Dirichlet Form}

In this section we want to explore some of the consequences of assuming that the density $f_{N}$ satisfies

$$
E^{f_{N}}\left[\frac{1}{N} \sum V\left(N\left(x_{i}-x_{j}\right)\right)\right] \leqq B
$$

and

$$
\frac{1}{2} \int\left|\nabla f_{N}\right|^{2} \frac{1}{f_{N}} d \mu_{N}=I_{N}\left(f_{N}\right) \leqq D N .
$$

The basic estimate uses integration by parts for any "suitable" test function $u=u\left(x_{1}, \ldots, x_{N}\right)$

$$
\begin{aligned}
\left|E^{f_{N}}\left[L_{N} u\right]\right| & =\left|\int\left(L_{N} u\right) f_{N} d \mu_{N}\right| \\
& =\left|\frac{1}{2} \int\left\langle\nabla u, \nabla f_{N}\right\rangle d \mu_{N}\right| \\
& \leqq\left(\frac{1}{2} \int|\nabla u|^{2} f_{N} d \mu_{N}\right)^{1 / 2}\left(\frac{1}{2} \int\left|\nabla f_{N}\right|^{2} \frac{1}{f_{N}} d \mu_{N}\right)^{1 / 2} \\
& \leqq\left(\frac{1}{2} \int|\nabla u|^{2} f_{N} d \mu_{N}\right)^{1 / 2}(D N)^{1 / 2}
\end{aligned}
$$

Of course such an estimate is only as good as the test functions $u$ that we can find to use in the estimate. The following class of test functions will be particularly useful:

$$
u_{N}\left(x_{1}, \ldots, x_{N}\right)=\sum_{i, j} \mathscr{G}_{N, \varepsilon, \lambda}\left(x_{\imath}-x_{j}\right),
$$

where $\mathscr{G}_{N, \varepsilon, \lambda}(x)$ is a function on $R$ that has sufficiently small support that it can be viewed as a function on $S$. For each fixed $\lambda$ and $\varepsilon$ we will have a choice of $u_{N}$ made for all sufficiently large $N$. The function $\mathscr{G}_{N, \varepsilon, \lambda}(x)$ is given by

$$
\mathscr{G}_{N, \varepsilon, \lambda}(x)=\int_{-\infty}^{x} G_{N, \varepsilon, \lambda}(y) d y
$$

and

$$
G_{N, \varepsilon, \lambda}(x)=\int_{-\infty}^{x} g_{N, \varepsilon, \lambda}(y) d y,
$$

where $g_{N, \varepsilon, \lambda}(\cdot)$ is a function $R$ with support in a small interval around the origin, 
satisfying in addition

and

$$
\int_{-\infty}^{\infty} g_{N, \varepsilon, \lambda}(y) d y=0
$$

$$
\int_{-\infty}^{\infty} y g_{N, \varepsilon, \lambda}(y) d y=0
$$

so that $G$ and $\mathscr{G}$ have the same support as $g$. The function $g_{N, \varepsilon, \lambda}(y)$ will be of the form

$$
g_{N, \varepsilon, \lambda}(y)=(N \varepsilon) g(N \varepsilon y)-\lambda g(\lambda y),
$$

where $g$ is a nonnegative, smooth, symmetric function with compact support with total integral 1 ,

$$
\int g(y) d y=1 .
$$

Clearly if $\lambda$ is big enough and $\varepsilon>0$ is arbitrary then for sufficiently large $N, u_{N}\left(x_{1}, \ldots, x_{N}\right)$ is well defined.

In order to use the integration by parts estimate (5.3) we have to compute $\mathscr{L}_{N} u_{N}$ and $\left|\nabla u_{N}\right|^{2}$,

$$
\begin{aligned}
\mathscr{L}_{N} u_{N}= & N \varepsilon \sum g\left(N \varepsilon\left(x_{i}-x_{j}\right)\right)-\lambda \sum g\left(\lambda\left(x_{i}-x_{j}\right)\right) \\
- & 2 N \sum_{i}\left(\sum_{j}\left[G\left(N \varepsilon\left(x_{i} x_{j}\right)\right)-G\left(\lambda\left(x_{i}-x_{j}\right)\right)\right]\right) \\
& \cdot\left(\sum_{k} V^{\prime}\left(N\left(x_{i}-x_{k}\right)\right)\right), \\
\left|\nabla u_{N}\right|^{2}= & 4 \sum_{i}\left|\sum_{j}\left[G\left(N \varepsilon\left(x_{i}-x_{j}\right)\right)-G\left(\lambda\left(x_{i}-x_{j}\right)\right)\right]\right|^{2} .
\end{aligned}
$$

One can use the fact that $V^{\prime}$ is an odd function to rewrite (5.11) in the form

$$
\begin{aligned}
\mathscr{L}_{N} u_{N}= & N \varepsilon \sum g\left(N \varepsilon\left(x_{i}-x_{j}\right)\right)-\lambda \sum g\left(\lambda\left(x_{i}-x_{j}\right)\right) \\
& -N \sum_{i, j, k}\left[G\left(N \varepsilon\left(x_{i}-x_{j}\right)\right)-G\left(N \varepsilon\left(x_{k}-x_{j}\right)\right)\right. \\
& \left.-G\left(\lambda\left(x_{i}-x_{j}\right)\right)+G\left(\lambda\left(x_{k}-x_{j}\right)\right)\right] V^{\prime}\left(N\left(x_{i}-x_{k}\right)\right) .
\end{aligned}
$$

Let us take the special case of $\varepsilon=1$ and $\lambda=1$. We assume that the support of $g$ is small enough that $\lambda=1$ works. Otherwise we can take any value $\lambda_{0}$ for $\lambda$.

We can write inequality (5.3) in the form

$$
\left|E^{f_{N}} \frac{1}{N^{2}} \mathscr{L}_{N} \mathscr{U}_{N}\right| \leqq \frac{D^{1 / 2}}{N^{3 / 2}}\left[E^{f_{N}}\left\{\frac{1}{2}\left|\nabla u_{N}\right|^{2}\right\}\right]^{1 / 2},
$$

with $\varepsilon=1$, the first term on the right-hand side of (5.11) is

$$
E^{f_{N}}\left\{\frac{1}{N} \sum g\left(N\left(x_{i}-x_{j}\right)\right)\right\}
$$


which is uniformly bounded because of (5.1) and Lemma 4.2. The second term is

$$
E^{f_{N}}\left\{\frac{1}{N^{2}} \sum g\left(x_{i}-x_{j}\right)\right\}
$$

which is bounded by a bound for $g$. The term

$$
\begin{aligned}
& \frac{1}{N} \sum_{i, j, k}\left[G\left(x_{i}-x_{j}\right)-G\left(x_{k}-x_{j}\right)\right] V^{\prime}\left(N\left(x_{i}-x_{k}\right)\right) \\
& \quad \leqq \frac{C}{N} \sum_{i, j, k}\left|x_{i}-x_{k}\right|\left|V^{\prime}\left(N\left(x_{i}-x_{k}\right)\right)\right|
\end{aligned}
$$

by using a bound on the derivative of $G$. We can use the function $\psi(x)=-x V^{\prime}(x)$ and write this term as

$$
\frac{C}{N^{2}} \sum_{i, j, k} \psi\left(N\left(x_{\imath}-x_{k}\right)\right) \leqq \frac{C}{N} \sum_{i, k} \psi\left(N\left(x_{i}-x_{k}\right)\right),
$$

which is bounded by (5.1) and Lemma 4.2. It is easy to estimate

$$
\left|\nabla u_{N}\right|^{2} \leqq C N^{3}
$$

by using a bound for $G$. Since in the inequality (5.3) every term has been bounded except for a single term we obtain

$$
E^{f_{N}}\left\{\frac{1}{N} \sum_{i, j, k}\left[G\left(N\left(x_{k}-x_{j}\right)\right)-G\left(N\left(x_{\imath}-x_{j}\right)\right)\right] V^{\prime}\left(N\left(x_{i}-x_{k}\right)\right)\right\} \leqq C,
$$

where $C$ is a bound depending only on $B$ and $D$. The function $F(x-y, y-z)$ $=V^{\prime}(x-z)[G(z-y)-G(x-y)]$ is nonnegative and one can check easily that it is possible to pick $g$ in such a manner that

$$
F(x-y, y-z) \geqq \psi(x-z) \psi(x-y) \text {. }
$$

From (5.14) and (5.15) we obtain

$$
\begin{aligned}
E^{f_{N}}\left\{\left(\frac{1}{N} \sum_{i, j} \psi\left(N\left(x_{i}-x_{j}\right)\right)\right)^{2}\right\} & \leqq E^{f_{N}}\left\{\frac{1}{N} \sum_{i}\left(\sum_{j} \psi\left(N\left(x_{i}-x_{j}\right)\right)\right)^{2}\right\} \\
& =E^{f_{N}}\left\{\frac{1}{N} \sum_{i, j, k} \psi\left(N\left(x_{i}-x_{j}\right)\right) \psi\left(N\left(x_{i}-x_{k}\right)\right)\right\} \\
& \leqq E^{f_{N}}\left\{\frac{1}{N} \sum_{i, j, k} F\left(N\left(x_{i}-x_{j}\right), N\left(x_{j}-x_{k}\right)\right)\right\} \\
& \leqq C .
\end{aligned}
$$

In other words (5.1) alone is sufficient to provide a bound for

$$
E^{f_{N}}\left\{\frac{1}{N} \sum \psi\left(N\left(x_{i}-x_{j}\right)\right)\right\}
$$


while (5.1) and (5.2) provide a bound for

$$
E^{f_{N}}\left\{\frac{1}{N} \sum \psi\left(N\left(x_{i}-x_{j}\right)\right)\right\}^{2} \text {. }
$$

We have therefore proved Lemma 5.1.

Lemma 5.1. There is a constant $C$ depending only on $B$ and $D$ such that

$$
\begin{aligned}
& E^{f_{N}}\left\{\frac{1}{N} \sum \psi\left(N\left(x_{i}-x_{j}\right)\right)\right\}^{2} \leqq C, \\
& E^{f_{N}}\left\{\frac{1}{N} \sum_{i}\left[\sum_{j} \psi\left(N\left(x_{i}-x_{j}\right)\right)\right]^{2}\right\} \leqq C .
\end{aligned}
$$

\section{Compactness}

In this section we will establish Theorem 3.1. Since the state space $M_{1}(S)$ is compact we need only establish estimates of modulus of continuity to appeal to Prohorov's theorem. It is therefore enough to prove

Lemma 6.1. For any smooth function $J$ on $S$, and $\varepsilon>0$,

$$
\lim _{\delta \rightarrow 0} \limsup _{N \rightarrow \infty} Q_{N}\left[\sup _{\substack{0 \leqq s<t \leqq T \\|s-t| \leqq \delta}}\left|\frac{1}{N} \sum J\left(x_{i}(s)\right)-\frac{1}{N} \sum J\left(x_{i}(t)\right)\right| \geqq \varepsilon\right]=0 .
$$

Proof. Let us take $s<t$. Then

$$
\begin{aligned}
& \frac{1}{N} \sum J\left(x_{i}(t)\right)-\frac{1}{N} \sum J\left(x_{i}(s)\right) \\
& =\frac{1}{2 N} \sum \int_{s}^{t} J^{\prime \prime}\left(x_{i}(\sigma)\right) d \sigma+\frac{1}{N} \int_{s}^{t} \sum J^{\prime}\left(x_{i}(\sigma)\right) d \beta_{i}(\sigma) \\
& \left.\quad-\int_{s}^{t} \sum V^{\prime}\left(N\left(x_{i}(\sigma)\right)-x_{j}(\sigma)\right)\right) J^{\prime}\left(x_{i}(\sigma)\right) d \sigma \\
& =\eta_{1}+\eta_{2}+\eta_{3} .
\end{aligned}
$$

The first term is clearly bounded by

$$
\left|\eta_{1}\right| \leqq \frac{1}{2} C(t-s)
$$

where $C$ is a bound for $J^{\prime \prime}$.

The second term is a Martingale term and by Doob's inequality

$$
\begin{aligned}
& E \sup _{0 \leqq t \leqq T}\left|\frac{1}{N} \sum \int_{0}^{t} J^{\prime}\left(x_{i}(\sigma)\right) d \beta_{i}(\sigma)\right|^{2} \\
& \quad \leqq C \exp _{0 \leqq t \leqq T} E\left|\frac{1}{N} \sum \int_{0}^{t} J^{\prime}\left(x_{i}(\sigma)\right) d \beta_{i}(\sigma)\right|^{2}
\end{aligned}
$$




$$
\begin{aligned}
& =C \frac{1}{N^{2}} \int_{0}^{T} \sum\left|J^{\prime}\left(x_{i}(\sigma)\right)\right|^{2} d \sigma \\
& \leqq \frac{C \cdot C_{1}^{2} T}{N},
\end{aligned}
$$

where $C_{1}$ is a bound for $J^{\prime}$.

The third term can be estimated by estimating the integrand

$$
\begin{aligned}
& \sum_{i j} V^{\prime}\left(N\left(x_{i}(\sigma)-x_{j}(\sigma)\right)\right) J^{\prime}\left(x_{i}(\sigma)\right) \\
& =\sum_{i j} V^{\prime}\left(N\left(x_{i}(\sigma)-x_{j}(\sigma)\right)\right)\left(J^{\prime}\left(x_{i}(\sigma)\right)-J^{\prime}\left(x_{j}(\sigma)\right)\right) \\
& \leqq C \sum_{i, j}\left|V^{\prime}\left(N\left(x_{i}(\sigma)-x_{j}(\sigma)\right)\right)\right|\left|x_{i}(\sigma)-x_{j}(\sigma)\right|,
\end{aligned}
$$

where $C$ is a bound for $J^{\prime \prime}$. Recalling that $\psi(z)=-z V^{\prime}(z)$ we can estimate

$$
\left|\eta_{3}\right| \leqq C \int_{s}^{t} \frac{1}{N} \sum \mid \psi\left(N\left(x_{\imath}(\sigma)-x_{j}(\sigma)\right) \mid d \sigma\right.
$$

One can then complete the proof of Lemma 4.1 if we show the following estimate:

$$
\sup _{N} E^{Q_{N}} \int_{0}^{T}\left[\frac{1}{N} \sum \psi\left(N\left(x_{i}(\sigma)-x_{j}(\sigma)\right)\right)\right]^{2} d \sigma<\infty .
$$

This follows from Lemma 5.1.

\section{Local Gibbs States}

In establishing the hydrodynamic scaling limit one of the problems we have to deal with is expressing average microscopic quantities like $\frac{1}{N} \sum \psi\left(N\left(x_{i}-y_{j}\right)\right) U\left(x_{i}\right)$ purely in terms of the macroscopic density function. This is of course possible only if the configuration $x_{1}, \ldots, x_{n}$ is suitably organized and we have to establish that during our stochastic dynamics most of the time the configurations are suitably organized with a very high probability.

To make this precise let us fix a value of $l$ and consider a functional $H(\omega)$ which is a bounded and continuous functional of the configuration of points in the interval $(-l, l)$. We can view $H(\omega)$ as a functional of a point process on $R$, which depends however only on the configuration of points in $(-l, l)$. If we have a set $\left(x_{1}, \ldots, x_{N}\right)$ of $N$ points in $S$, for any given $x$, we can look at the points $x_{i}:\left|x_{i}-x\right|<\frac{1}{4}$ and consider the points $N\left(x_{i}-x\right)$ on the line $(-\infty, \infty)$ obtained from the original $\left(x_{1}, \ldots, x_{N}\right)$ on $S$. The resulting point process on $R$, while it is somewhat arbitrary due to the cutoff at the edges namely around $\pm \frac{N}{4}$, for every $l$ the configuration is natural and well defined on $[-l, l]$, provided $N$ is large enough. We can evaluate our functional $H(\omega)$ at this configuration, which we denote by $\omega_{N}^{x}$ and obtain a value $H\left(\omega_{N}^{x}\right)$. 
We are interested in the quantity

$$
\xi_{N}\left(x_{1}, \ldots, x_{N}\right)=\int_{S} H\left(\omega_{N}^{x}\right) U(x) d x
$$

Corresponding to the set $x_{1}, \ldots, x_{N}$ we have the empirical distribution

$$
\alpha_{N}(d x)=\frac{1}{N}\left(\delta_{x_{1}}+\ldots+\delta_{x_{N}}\right)
$$

We pick a mollifier $h(x)$ with compact support, i.e. a nonnegative function $h(x)$ with $\int h(x) d x=1$, and assuming that the support of $h$ is contained in $\left[-\frac{1}{2}, \frac{1}{2}\right]$, we can lift $h$ as a function on $S$, and for $\lambda \geqq 1, \lambda h(\lambda x)$ is a mollifier on $S$ as well. We can then pick for $\lambda \geqq 1$, a natural version of local density for the configuration $\left(x_{1}, \ldots, x_{N}\right)$, by

$$
\begin{aligned}
\rho_{\lambda}(x) & =\frac{\lambda}{N} \sum h\left(\lambda\left(x_{i}-x\right)\right) \\
& =\left(h_{\lambda} * \alpha_{N}\right)(x), \quad \text { where } \\
h_{\lambda} & =\lambda h(\lambda x) .
\end{aligned}
$$

For a one dimensional system, there are no phase transitions and we have a unique Gibbs state $P_{\rho}$ viewed as a stationary point process with density $\rho$ that corresponds to the given pair interaction $V(x)$. Since we expect the arrangement of points near $x$ to be a random arrangement from $P_{\rho}$ with $\rho=\rho_{\lambda}$, by (7.1) we expect to replace $\xi_{N}\left(x_{1}, \ldots, x_{N}\right)$ by $\eta_{N, \lambda}\left(x_{1}, \ldots, x_{N}\right)$, where

$$
\eta_{N, \lambda}\left(x_{1}, \ldots, x_{N}\right)=\int_{S} \hat{H}\left(\rho_{\lambda}(x)\right) U(x) d x
$$

with

$$
\hat{H}(\rho)=E^{P \rho}[H(\omega)]
$$

The error between $\xi_{N}$ and $\eta_{N, \lambda}$ is expected to be small provided $\lambda$ is large and $N$ is large and $\left(x_{1}, \ldots, x_{N}\right)$ is a typical configuration from a nice distribution $f_{N} d \mu_{N}$.

Let us denote by $\mathscr{A}_{N, B, D}$ the set of probability densities $f_{N}$ on $S^{N}$ that satisfy

$$
\begin{gathered}
F^{f_{N}}\left\{\frac{1}{N} \sum V\left(N\left(x_{i}-x_{j}\right)\right)\right\} \leqq B, \\
\frac{1}{2} \int \frac{\left|\nabla f_{N}\right|^{2}}{f_{N}} d \mu_{N} \leqq D N .
\end{gathered}
$$

Because of translation invariance of the expressions (7.5) and (7.6), if we replace $f_{N}$ by

$$
\hat{f}_{N}\left(x_{1}, \ldots, x_{N}\right)=\int_{S} f_{N}\left(x_{1}+a, \ldots, x_{N}+a\right) d a
$$

due to linearity in the case of (7.5) and convexity in the case of (7.6), $\hat{f}_{N}$ will satisfy the inequalities (7.5) and (7.6). In other words, if $f_{N} \in \mathscr{A}_{N, B, D}$ so does $\hat{f}_{N}$. Let us denote by $\mathscr{A}_{N, B, D}^{0}$, the translation invariant densities in $\mathscr{A}_{N, B, D}$. Our goal is to establish that for each fixed $l$ and $H(\cdot)$ 


\section{Theorem 7.1.}

$$
\lim _{\lambda \rightarrow \infty} \limsup _{N \rightarrow \infty} \sup _{f_{N} \in \mathscr{A}_{N, B, D}} E^{f_{N}}\left|\xi_{N}-\eta_{N, \lambda}\right|=0 .
$$

Since $P_{\rho}$ depends continuously on $\rho, \hat{H}(\rho)$ is continuous in $\rho$ and we can establish Theorem 7.1 in two steps.

\section{Theorem 7.2.}

and

$$
\lim _{\varepsilon \rightarrow 0} \limsup _{N \rightarrow \infty} \sup _{f_{N} \in \mathscr{L}_{N, B, D}} E^{f_{N}}\left|\xi_{N}-\eta_{N, N_{\varepsilon}}\right|=0
$$

\section{Theorem 7.3.}

$$
\lim _{\substack{\varepsilon \rightarrow 0 \\ \lambda \rightarrow \infty}} \limsup _{N \rightarrow \infty} \sup _{f_{N} \mathscr{A}_{N, B, D}} E^{f_{N}} \int_{S}\left|\rho_{\lambda}(x)-\rho_{\varepsilon N}(x)\right| d x=0 .
$$

Remark. In view of the comment made earlier about translation invariance in Theorems 7.1, 7.2 and 7.3 we can in the proof replace $\mathscr{A}_{N, B, D}$ by $\mathscr{A}_{N, B, D}^{0}$.

We can write

$$
\begin{aligned}
& E^{f_{N}}\left|\int H\left(\omega_{N}^{x}\right) U(x) d x-\int \hat{H}\left(\rho_{\lambda}(x)\right) U(x) d x\right| \\
& \leqq E^{f_{N}}\left|\int\left[\frac{N}{2 a} \int_{|y-x| \leqq a / N} H_{0}\left(\omega_{N}^{x}\right) d x\right] U(y) d y-\int \hat{H}\left(\rho_{\lambda}(x)\right) U(x) d x\right| \\
& \quad+\|H\| E^{f_{N}} \int \frac{N}{2 a} \int_{|U-x| \leqq a / N}|U(y)-u(x)| d y d x .
\end{aligned}
$$

To fixed $a, H$ and any sequence $\lambda=\lambda_{N}$,

$$
\begin{aligned}
& \limsup _{N \rightarrow \infty} \sup _{f_{N} \in \mathscr{A}_{N, B, D}} E^{f_{N}}\left|\int H\left(\omega_{N}^{x}\right) U(x) d x-\int \hat{f}\left(\rho_{\lambda_{N}}(x)\right) U(x) d x\right| \\
& \leqq\|U\| \limsup _{N \rightarrow \infty} \sup _{f_{N} \in \mathscr{A}_{N, B, D}} E^{f_{N}}\left[\int d y\left|\frac{N}{2 a} \int_{|y-x| \leqq a / N} H\left(\omega_{N}^{x}\right) d x-\hat{H}\left(\rho_{\lambda_{N}}(y)\right)\right|\right]
\end{aligned}
$$

by assuming continuity of the function $U(x)$.

If we now replace $f_{N}$ by $\hat{f}_{N}$ then

$$
\begin{aligned}
E^{f_{N}} & {\left[\int d y \mid \frac{N}{2 a} \int_{|y-x| \leqq a / N} H\left(\omega_{N}^{x}\right) d x-\hat{H}\left(\rho_{\lambda_{N}}(y) \mid\right]\right.} \\
= & E^{f_{N}}\left[\int d y \mid \frac{N}{2 a} \int_{|y-x| \leqq a / N} H\left(\omega_{N}^{x}\right) d x-\hat{H}\left(\rho_{\lambda_{N}}(y) \mid\right]\right. \\
= & E^{f_{N}}\left[\left|\frac{N}{2 a} \int_{|x| \leqq a / N} H\left(\omega_{N}^{x}\right) d x-\hat{H}\left(\rho_{\lambda_{N}}(0)\right)\right|\right] .
\end{aligned}
$$

The proof of Theorem 7.2 can therefore be reduced to the proof of the following theorem. 


\section{Theorem 7.4.}

$$
\varlimsup_{\varepsilon \rightarrow 0} \limsup _{N \rightarrow \infty} \sup _{f_{N} \in \mathscr{A}_{N, B, D}^{0}} E^{f_{N}}\left|\frac{N \varepsilon}{2} \int_{|x| \leqq 1 / N \varepsilon} H\left(\omega_{N}^{x}\right) d x-\hat{H}\left(\rho_{N_{\varepsilon}}(0)\right)\right|=0 .
$$

The proof of Theorem 7.4 proceeds as follows.

The computation of $\int_{|x| \leq 1 / N \varepsilon} H\left(\omega_{N}^{x}\right) d x$ and $\rho_{N_{\varepsilon}}(0)$ requires only a knowledge of the location of the particles from among $x_{1}, \ldots, x_{N}$ that belong to some interval of the form $|x| \leqq \frac{1}{N \varepsilon}+\frac{l_{0}}{N}$, where $\left[-l_{0}, l_{0}\right]$ is such that $H(\omega)$ depends only on the configuration there. So if we project $f_{N} d \mu_{N}$ onto the configurations in such an interval and expand the interval by a factor of $N$, we will get a set $\mathscr{B}_{N, B, D, \varepsilon}^{0}$ of point processes on the interval $|x| \leqq \frac{1}{\varepsilon}+l_{0}$. Theorem 7.4 reduces to proving that

$$
\lim _{\varepsilon \rightarrow 0} \limsup _{N \rightarrow \infty} \sup _{\gamma \in \mathscr{F}_{N, B, D, \varepsilon}^{N}} E^{\gamma}\left|\frac{\varepsilon}{2} \int_{-1 / \varepsilon}^{1 / \varepsilon} H\left(\omega^{x}\right) d x-\hat{H}\left(\rho_{\varepsilon}(0)\right)\right|=0 .
$$

Let us note that because of translation invariance and blowup by a factor of $N$, the local density of particles is normalized at 1 . Therefore the collection $\mathscr{B}_{N, B, D, \varepsilon}^{0}$ of point processes on $\left[-\left(\frac{1}{\varepsilon}+l_{0}\right),\left(\frac{1}{\varepsilon}+l_{0}\right)\right]$ is compact as $N \rightarrow \infty$ and we denote by $\widehat{\mathscr{B}}_{B, D, \varepsilon}$ the set of limit points. Verifying (7.7) reduces to verifying

$$
\lim _{\varepsilon \rightarrow 0} \sup _{\nu \in \mathscr{S}_{B, D, \varepsilon}} E^{\nu}\left[\left|\frac{\varepsilon}{2} \int_{-1 / \varepsilon}^{1 / \varepsilon} H\left(\omega^{x}\right) d x-\hat{H}\left(\rho_{\varepsilon}(0)\right)\right|\right]=0 .
$$

In the appendix we have introduced the collection of Gibbs measure with boundary condition $\omega$ and particle number $n$ on the interval $[-l, l]$. Let us denote the convex hull of these measures with $n$ and $\omega$ varying by $\Gamma$. The subset with the average particle density less than or equal to 1 will be denoted by $\Gamma_{l}^{(1)}$. According to Theorem 10.3 of the appendix,

$$
\lim _{l \rightarrow \infty} \sup _{v \in \Gamma_{l+l_{0}}^{(1)}} E^{\nu}\left[\left|\frac{1}{2 l} \int_{-l}^{l} H\left(\omega^{x}\right) d x-\hat{H}\left(\rho_{l}(0)\right)\right|\right]=0,
$$

and we will complete the proof of (7.8) by establishing

Lemma 7.5. For any $B, D$ and $\varepsilon>0$

$$
\widehat{\mathscr{B}}_{B, D, \varepsilon} \subset \Gamma_{1 / \varepsilon+l_{0}}^{(1)} .
$$

Proof. Since the density can only drop under the weak limit it is clear that it is sufficient to prove $\mathscr{\mathscr { B }}_{B, D, \varepsilon} \subset \Gamma_{l_{0}+1 / \varepsilon}$.

Let us assume that $V$ is supported on some $\left[-c_{0}, c_{0}\right]$ and look at the set of limit points of the point process on a slightly larger interval of the form $\left[-\left(\frac{1}{\varepsilon}+l_{0}+c_{0}\right), \frac{1}{\varepsilon}+l_{0}+c_{0}\right]$ in the expanded scale. Let $\gamma$ be any limit point. We want to characterize the restriction of $v$ as a point process on 
$\left[-\left(\frac{1}{\varepsilon}+l_{0}\right),\left(\frac{1}{\varepsilon}+l_{0}\right)\right]$ which is a member of $\Gamma_{l_{0}+1 / \varepsilon}$. Let us consider a diffusion on the configuration space of point processes on the interval $\left[-\left(\frac{1}{\varepsilon}+l_{0}+c_{0}\right), \frac{1}{\varepsilon}+l_{0}+c_{0}\right]$ under which the particles in $\left[-\left(\frac{1}{\varepsilon}+l_{0}\right)\right.$, $\left.\left(\frac{1}{\varepsilon}+l_{0}\right)\right]$ diffuse with reflecting boundary conditions at the end point and those outside $\left[-\left(\frac{1}{\varepsilon}+l_{0}\right),\left(\frac{1}{\varepsilon}+l_{0}\right)\right]$ stay put. The generator is given by

$$
L=\frac{1}{2} \sum \frac{\partial^{2}}{\partial x_{i}^{2}}-\sum_{i, j} V^{\prime}\left(x_{\imath}-x_{j}\right) \frac{\partial}{\partial x_{i}}-\frac{1}{2} \sum_{i, \alpha} V^{\prime}\left(x_{i}-y_{\alpha}\right) \frac{\partial}{\partial x_{i}},
$$

where $x_{1}, \ldots, x_{n}$ are the particles inside and $\left\{y_{\alpha}\right\}, \ldots$ are the particles outside. The outside configuration $\omega$ and the number $n$ of particles inside do not change and for this diffusion $L$ the family $\mu_{n, l^{\prime}}^{\omega}$ with $l^{\prime}=\left(\frac{1}{\varepsilon}+l_{0}\right)$ is precisely the set of extremal invariant measures. Therefore all we need to show is that $v$ is invariant for the diffusion $L$.

Let us take a function $u\left(x_{1}, \ldots, x_{n}, b\right)$ of the form $u\left(x_{1}, \ldots, x_{n}\right) \phi(b)$ with a smooth $u$, satisfying Neumann boundary conditions. Strictly speaking $u\left(x_{1}, \ldots, x_{n}, b\right)$ is not a continuous function viewed as a function on configurations in $\left[-\left(\frac{1}{\varepsilon}+l+k_{0}\right), \frac{1}{\varepsilon}+l+k_{0}\right]$ because of the distinction between outside and inside variables. Its discontinuity points are on the set where a particle sits exactly on the boundary. These have probability zero because the homogeneity in space makes any local density at most one in the limit. So we can calculate

$$
\begin{aligned}
\int_{A_{n}} L u d v & =\lim _{N \rightarrow \infty} \int_{A_{n}} L u d v_{N} \\
& =\lim _{N \rightarrow \infty} \int_{A_{n, N}} \hat{L}_{N} \hat{u}_{N} f_{N} d \mu_{N},
\end{aligned}
$$

where $f_{N} d \mu_{N}$ is the measure on $S^{N}$ which produced the $v_{N}$ by restricting in the set $\mathscr{B}_{N, B, D, \varepsilon^{\prime}}^{0}$ for some choice of $\varepsilon^{\prime} . A_{n}$ is the set with $n$ points in $\left[-\left(\frac{1}{\varepsilon}+l_{0}\right),\left[\frac{1}{\varepsilon}+l_{0}\right]\right]$ and $A_{n, N}$ is the set with $n$ points in $\left[-\frac{\left(\frac{1}{\varepsilon}+l_{0}\right)}{N}, \frac{\left(\frac{1}{\varepsilon}+l_{0}\right)}{N}\right]$.

An elementary calculation using integration by parts yields

$$
\int_{A n, N} \hat{L}_{N} \hat{u}_{N} f_{N} d \mu_{N}=\frac{1}{2 N^{2}} \int_{A n, N} \sum^{\prime} \frac{\partial \hat{u}_{n}}{\partial x_{i}} \cdot \frac{\partial f_{N}}{\partial x_{i}} d \mu_{N}
$$

where $\Sigma^{\prime}$ is the summation over those variables $x_{1}, \ldots, x_{n}$ inside $\left[-\frac{\left(\frac{1}{\varepsilon}+l_{0}\right)}{N}, \frac{\left(\frac{1}{\varepsilon}+l_{0}\right)}{N}\right]$. The factor $\frac{1}{N^{2}}$ comes from the stretching. $\frac{\partial \hat{u}_{n}}{\partial x_{\imath}}$ are of 
order $N$ and therefore we only have to prove

$$
\sup _{N} \frac{1}{2} \int_{A_{n, N}} \sum^{\prime}\left(\frac{\partial f_{N}}{\partial x_{i}}\right)^{2} \cdot \frac{1}{f_{N}} d \mu_{N}<\infty .
$$

We can calculate by translation invariance

$$
\begin{aligned}
\int \frac{1}{2} \sum^{\prime}\left(\frac{\partial f_{N}}{\partial x_{i}}\right)^{2} \frac{1}{f_{N}} d \mu_{N} & =\frac{2\left(\frac{1}{\varepsilon}+l_{0}\right)}{N} \int \frac{1}{2} \sum\left(\frac{\partial f_{N}}{\partial \mathrm{x}_{i}}\right)^{2} \frac{1}{f_{N}} d \mu_{N} \\
& \leqq \frac{2\left(\frac{1}{\varepsilon}+l_{0}\right)}{N} \cdot N D \\
& =2\left(\frac{1}{\varepsilon}+l_{0}\right) D
\end{aligned}
$$

and we are done.

This proves Theorem 7.2.

We now turn to the proof of Theorem 7.3. We have to carry out some construction. Given any set $\left(x_{1}, \ldots, x_{N}\right)$ of $N$ points in $S$, we have earlier associated the empirical distribution

$$
\alpha_{N}(d x)=\frac{1}{N}\left[\delta_{x_{1}}+\ldots+\delta_{x_{N}}\right]
$$

and a mollified density

$$
\rho_{\lambda}(x)=\frac{\lambda}{N} \sum h\left(\lambda\left(x-x_{i}\right)\right) .
$$

For most configurations the density $\rho_{\varepsilon N}(x)$ can be highly oscillatory and $\int \hat{H}\left(\rho_{\varepsilon N}(x)\right) d x$ and $\int \hat{H}\left(\rho_{\lambda}(x)\right) d x$ for fixed $\varepsilon$ and $\lambda$ can be very far apart. To rule this out we plan to calculate the Young measure associated with the functions $\rho_{\varepsilon N}(\cdot)$ and show that for $N$ large and $\varepsilon$ small, these are nearly degenerate. Corresponding to a mollified density $\rho_{\lambda}(x)$ we can define a probability measure $\pi$ on $S \times R^{+}$by the relation

$$
\int F(x, \rho) \pi(d x, d \rho)=\int_{S} F\left(x, \rho_{\lambda}(x)\right) d x
$$

$\pi$ clearly enjoys the properties

$$
\begin{aligned}
& \int \rho \pi(d x, d \rho)=\int \rho_{\lambda}(x) d x=1, \\
& \int F(x) \pi(d x, d \rho)=\int F(x) d x,
\end{aligned}
$$

or $\pi$ projects to Lebesgue measure on $S$. If we denote by $\mathscr{M}$ the space of probability measures on $S \times R^{+}$, then through $\pi$ and $\rho_{\lambda}$ we map $x_{1}, \ldots, x_{N}$ into $\mathscr{M}$. We already have a map $\mathscr{L}_{n}$ that maps $\left(x_{1}, \ldots, x_{n}\right)$ into $M_{1}(S)$ by

$$
\alpha_{N}(d x)=\frac{1}{N}\left[\delta_{x_{1}}+\ldots+\delta_{\alpha_{N}}\right] .
$$


We now take both the maps and map $\left(x_{1}, \ldots, x_{N}\right)$ into the pair $\alpha_{N}, \pi$, a point in $M_{1}(S) \times \mathscr{M}$. With the choice of $\lambda=N \varepsilon$, this produces an induced probability measure $\hat{Q}_{N, \varepsilon}$ on $M_{1}(S) \times \mathscr{M}$ for each $f_{N}$ from $\mathscr{A}_{N, B, D}$ and choice of $\varepsilon$. The range of such $\hat{Q}_{N, \varepsilon}$ will be denoted by $\hat{\mathscr{A}}_{N, B, D, \varepsilon}$, their limit points as $N \rightarrow \infty$ by $\hat{\mathscr{A}}_{B, D, \varepsilon}$ and the limit points of these as $\varepsilon \rightarrow 0$ by $\hat{\mathscr{A}}_{B, D}$. Our goal is to prove Theorem 7.3 and the main step is

Theorem 7.6. Let $\hat{Q} \in \hat{\mathscr{A}}_{B, D}$. Then for almost all $(\alpha, \pi)$ with respect to $\hat{Q}$,

and

$$
\alpha(d x)=\rho(x) d x
$$

$$
\pi(d x, d \rho)=d x \delta_{\rho(x)}(d \rho)
$$

for some function $\rho(x)$ in $L_{1}(S)$, i.e. the young measures are trivial.

We will reach Theorem 7.6 by a series of lemmas:

Lemma 7.7. Let $\hat{Q} \in \hat{\mathscr{A}}_{B, D}$. Then $\hat{Q}$ has the property

and in fact

$$
\hat{Q}[\alpha: \alpha(d x)=\rho(x) d x \text { for some } \rho]=1 \text {, }
$$

$$
E^{Q}\left[\int_{S} \rho^{2}(x) d x\right] \leqq C \cdot B
$$

where $C$ is a universal constant.

Proof. From Lemma 4.2 we can conclude that

$$
E^{Q} \int_{S} \lambda W(\lambda(x-y)) \alpha(d \alpha) \alpha(d y) \leqq\|W\| C B,
$$

where $W$ is a nonnegative function of integral 1 . Since the bound is uniform in $\lambda$ we can let $\lambda \rightarrow \infty$ and establish Lemma 7.7 by Fatou's lemma.

Lemma 7.8. For any $Q_{\varepsilon}$ in $\hat{\mathscr{A}}_{B, D, \varepsilon}$,

$$
\begin{gathered}
Q_{\varepsilon}\left[(\alpha, \pi): \int \rho \pi(d x, d \rho)=1\right]=1, \\
Q_{\varepsilon}\left[(\alpha, \pi): \pi(d x, d \rho)=d x \pi_{x}(d \rho)\right]=1, \\
Q_{\varepsilon}\left[(\alpha, \pi): \alpha(d x)=\rho(x) d x \text { with } \rho(x)=\int \rho \pi_{x}(d \rho)\right]=1 .
\end{gathered}
$$

Proof.

$$
\begin{aligned}
E^{Q_{N, \varepsilon}} \int \rho^{2} \pi(d x, d \rho) & =E^{f_{N}} \int_{S} \rho_{\varepsilon N}^{2}(x) d x \\
& =E^{f_{N}} \int_{S}\left|\varepsilon \sum h\left(\varepsilon N\left(x_{i}-x\right)\right)\right|^{2} d x \\
& =\frac{\varepsilon}{N} E^{f_{N}} \sum W\left(\varepsilon N\left(x_{i}-x_{j}\right)\right) \\
& \leqq\|W\| B C \quad(\text { by Lemma 4.2) }
\end{aligned}
$$

Now (7.11) is a consequence of the unitform integrability (7.9). Relation (7.12) follows from (7.10). (7.13) is just the routine fact that the mean of the Young measures defines the function which is the weak limit. 
We now try to use the bound on the Dirichlet form and use inequality (5.3). The expression for $\mathscr{L}_{N} U_{N}$ given by (5.11) can be written as

$$
\frac{1}{N^{2}} \mathscr{L}_{N} U_{N}=T_{1}-T_{2}+T_{3}-T_{4}
$$

where

$$
\begin{aligned}
& T_{1}=\frac{\delta}{N} \sum g\left(N \delta\left(x_{i}-x_{j}\right)\right), \\
& T_{2}=\frac{\lambda}{N^{2}} \sum g\left(\lambda\left(x_{i}-x_{j}\right)\right) \\
& T_{3}=\frac{1}{N} \sum_{i, j, k}\left[G\left(N \delta\left(x_{k}-x_{j}\right)\right)-G\left(N \delta\left(x_{i}-x_{j}\right)\right)\right] V^{\prime}\left(N\left(x_{i}-x_{k}\right)\right), \\
& \left.\left.T_{4}=\frac{1}{N} \sum_{i, j, k}\left[G\left(\lambda\left(x_{k}-x_{j}\right)\right)-G\right) \lambda\left(x_{i}-x_{j}\right)\right)\right] V^{\prime}\left(N\left(x_{i}-x_{k}\right)\right) .
\end{aligned}
$$

The inequality (5.3) takes the form

$$
E^{f_{N}}\left[T_{1}+T_{3}\right] \leqq E^{f_{N}}\left[T_{2}+T_{4}\right]+D^{1 / 2}\left(E^{f_{N}} T_{5}\right)^{1 / 2},
$$

where

$$
T_{5}=\frac{4}{N^{3}} \sum_{i}\left|\sum_{j} G\left(N \delta\left(x_{i}-x_{j}\right)\right)-G\left(\lambda\left(x_{i}-x_{j}\right)\right)\right|^{2} .
$$

Because $\left|G\left(N \delta\left(x_{t}-x_{j}\right)\right)-G\left(\lambda\left(x_{i}-x_{j}\right)\right)\right|$ is bounded by 1 and $G(N \delta x)-G(\lambda x)$ is supported on some interval of size $\lambda^{-1}$ we can bound

$$
\left|G\left(N \delta\left(x_{i}-x_{j}\right)\right)-G\left(\lambda\left(x_{i}-x_{j}\right)\right)\right| \leqq \chi\left(\lambda\left(x_{i}-x_{j}\right)\right),
$$

where $\chi(x)$ is a bounded nonnegative continuous function with compact support. Therefore in (7.15), $T_{5}$ can be replaced by

$$
T_{6}=\frac{4}{N^{2}} \sum_{i} \sum_{j} \chi\left(\lambda\left(x_{i}-x_{j}\right)\right) \text {. }
$$

We next want to chase the inequality (7.15) through subsequences to get a limit point of $\hat{Q}_{N, \varepsilon}$ in $\hat{\mathscr{A}}_{B, D, \varepsilon}$ and then again through limits finally to a limit $\hat{Q}$ in $\hat{\mathscr{A}}_{B, D}$.

We have

$$
\begin{aligned}
T_{1} & =\frac{\delta}{N} \sum g\left(N \delta\left(x_{i}-x_{j}\right)\right) \\
& =\delta \int_{S} \sum g\left(N \delta\left(x_{i}-x_{j}\right)\right) h\left(N\left(x_{i}-x\right)\right) d x \\
& =\delta \int_{S} H\left(\omega_{N}^{x}\right) d x,
\end{aligned}
$$

where

$$
H(\omega)=\sum g\left(\delta\left(x_{i}-x_{j}\right)\right) h\left(x_{i}\right) .
$$

If it were not for the fact that $H(\omega)$ is unbounded we could apply Theorem 7.2 
and we would have

with

$$
\lim _{N \rightarrow \infty} E^{f_{N}} T_{1}=E^{Q} \int d x\left[\int \hat{H}_{\delta}(\rho) \pi_{x}(d \rho)\right]
$$

$$
\begin{aligned}
\hat{H}_{\delta}(\rho) & =E_{\rho}\left[\delta \sum g\left(\delta\left(x_{i}-x_{j}\right)\right) h\left(x_{i}\right)\right] \\
& =\int \delta g(\delta(x-y)) h(x) R_{\rho}^{(2)}(d x, d y),
\end{aligned}
$$

where $R_{\rho}^{(2)}(d x, d y)$ is the two point correlation function relative to the Gibbs measure with density $\rho$. However since $H(\omega)$ is nonnegtive and continuous Fatou's lemma can be used to conclude

$$
E^{Q} \int_{S} d x\left[\int \hat{H}_{\delta}(\rho) \pi_{x}(d \rho)\right] \leqq \lim _{N \rightarrow \infty} E^{f_{N}} T_{1}
$$

We next look at $T_{3}$,

$$
\begin{aligned}
T_{3} & =\frac{1}{N} \sum_{i, j, k}\left[G\left(N \delta\left(x_{k}-x_{j}\right)\right)-G\left(N \delta\left(x_{i}-x_{j}\right)\right)\right] V^{\prime}\left(N\left(x_{i}-x_{k}\right)\right) \\
& =\int_{S} d x \sum\left[G \left(N \delta\left(x_{k}-x_{j}\right)-G\left(N \delta\left(x_{i}-x_{j}\right)\right] V^{\prime}\left(N\left(x_{i}-x_{k}\right)\right) h\left(N\left(x_{i}\right)\right.\right.\right. \\
& =\int_{S} H_{1}\left(\omega_{N}^{x}\right) d x
\end{aligned}
$$

where

$$
H_{1}(\omega)=\sum\left[G\left(\delta\left(x_{k}-x_{j}\right)\right)-G\left(\delta\left(x_{i}-x_{j}\right)\right)\right] V^{\prime}\left(x_{i}-x_{k}\right) h\left(x_{i}\right) .
$$

One can check that because of the monotonicity of $G$ and the fact that $x V^{\prime}(x) \leqq 0$ for all $x, H_{1}(\omega)$ is nonnegative. One can then obtain by exactly the same reasoning as above

$$
\left.E^{Q} \int_{S} d x\left[\int \hat{H}_{1, \delta}(\rho) \pi_{x}(d \rho)\right]\right] \leqq \lim _{N \rightarrow \infty} E^{f_{N}} T_{3},
$$

where

$$
\hat{H}_{1, \delta}(\rho)=\iint[G(\delta(z-y))-G(\delta(x-y))] V^{\prime}(x-y) h(x) R_{\rho}^{(3)}(d x, d y, d z),
$$

where $R_{\rho}^{(3)}(d x, d y, d z)$ is the three point correlation.

We now turn to $T_{2}$ :

$$
\begin{aligned}
E^{Q} \iint \lambda g(\lambda(x-y)) d \alpha(x) d \alpha(y) & =E^{Q} \iint \lambda g(\lambda(x-y)) \rho(x) \rho(y) d x d y \\
& =\lim _{N \rightarrow \infty} E^{f_{N}} T_{2} .
\end{aligned}
$$

As for $T_{4}$

$$
T_{4}=\frac{1}{N} \sum_{i, j, k}\left[G\left(\lambda\left(x_{k}-x_{j}\right)\right)-G\left(\lambda\left(x_{\imath}-x_{\jmath}\right)\right)\right] V^{\prime}\left(N\left(x_{\imath}-x_{k}\right)\right) .
$$

We know that $V^{\prime}\left(N\left(x_{i}-x_{k}\right)\right)=0$ unless $\left|x_{i}-x_{k}\right| \leqq C / N$. Therefore we can expand $G\left(\lambda\left(x_{k}-x_{j}\right)\right)-G\left(\lambda\left(x_{i}-x_{j}\right)\right)$ by Taylor's formula with remainder and 


$$
\left|G\left(\lambda\left(x_{k}-x_{j}\right)\right)-G\left(\lambda\left(x_{i}-x_{j}\right)\right)\right| \leqq \lambda g\left(\lambda\left(x_{k}-x_{j}\right)\right)\left(x_{k}-x_{i}\right)+\frac{C}{N}\left|x_{k}-x_{i}\right|
$$

where the error term depends only on $\lambda$.

We can now write

$$
\begin{gathered}
T_{4}=\frac{\lambda}{N^{2}} \sum g\left(\lambda\left(x_{i}-x_{j}\right)\right) \psi\left(N\left(x_{i}-x_{k}\right)\right)+\text { Error }, \\
E^{f_{N}}[\mid \text { Error } \mid] \leqq E^{f_{N}} \frac{C}{N^{2}} \sum_{i, j, k} \psi\left(N\left(x_{i}-x_{j}\right)\right) \\
\leqq \frac{C}{N} \text { by Lemma } 4.2 .
\end{gathered}
$$

We now try to evaluate

$$
\lim _{n \rightarrow \infty} E^{f_{N}}\left[\frac{\lambda}{N^{2}} \sum g\left(\lambda\left(x_{k}-x_{j}\right)\right) \psi\left(N\left(x_{i}-x_{k}\right)\right)\right] .
$$

The function $\lambda g(\lambda(x-y))$ on $S \times S$ can be approximated by functions of the form

$$
\sum_{r=1}^{l} a_{r}(x) b_{r}(y)
$$

uniformly on $S \times S$ and if we denote by

then

$$
\lambda g(\lambda(x-y))-\sum_{r=1}^{l} a_{r}(x) b_{r}(y)=c(x, y),
$$

$$
\begin{aligned}
E^{f_{N}}\left|\frac{1}{N^{2}} \sum c\left(x_{k}, x_{j}\right) \psi\left(N\left(x_{i}-x_{k}\right)\right)\right| & \leqq \frac{1}{N^{2}}\|C\| N E^{f_{N}}\left[\sum \psi\left(N\left(x_{i}-x_{j}\right)\right)\right] \\
& \leqq\|C\| \quad \text { by Lemma 4.2. }
\end{aligned}
$$

So the error is controlled uniformly in $N$. We can compute

$$
\begin{gathered}
\lim _{N \rightarrow \infty} E^{f_{N}} \frac{1}{N^{2}} \sum a\left(x_{k}\right) b\left(x_{j}\right) \psi\left(N\left(x_{i}-x_{k}\right)\right) \\
=\lim _{N \rightarrow \infty} E^{f_{N}}\left[\frac{1}{N} \sum b\left(x_{j}\right) \cdot \frac{1}{N} \sum a\left(x_{k}\right) \psi\left(N\left(x_{i}-x_{k}\right)\right)\right. \\
\left|\frac{1}{N} \sum a\left(x_{k}\right) \psi\left(N\left(x_{i}-x_{k}\right)\right)-\int_{S} a(y) \sum_{i, k} \psi\left(N\left(x_{i}-x_{k}\right)\right) h\left(N\left(x_{k}-y\right)\right)\right| \\
\leqq\left|\frac{1}{N} \sum \psi\left(N\left(x_{i}-x_{k}\right)\right) \int\left[a(y)-\left(x_{k}\right)\right] N h\left(N\left(x_{k}-y\right)\right) d y\right| \\
\leqq \frac{1}{N} \sum \psi\left(N\left(x_{i}-x_{k}\right) \cdot \varepsilon_{N},\right.
\end{gathered}
$$


where $\varepsilon_{N}$ depends only on the modulus of continuity of $a$ and goes to zero with $N$. Again by Lemma 4.2 we can control the error uniformly in $N$.

Now we can consider

$$
\lim _{N \rightarrow \infty} E^{f_{N}}\left\{\frac{1}{N} \sum b\left(x_{j}\right) \cdot \int_{S} a(y) \sum \psi\left(N\left(x_{i}-x_{k}\right)\right) h\left(N\left(x_{k}-y\right)\right) d y\right\},
$$

and working as before we should end up with

$$
\begin{aligned}
E^{Q} & \left\{\int_{S} b(x) \rho(x) d x \int_{S} a(y) \int_{R} \hat{H}_{3}(\rho) \pi_{y}(d \rho)\right\} \\
= & E^{Q}\left\{\iint b(x) a(y) \rho(x) \int_{R} \hat{H}_{3}(\rho) \pi_{y}(d \rho)\right\},
\end{aligned}
$$

where

$$
\hat{H}_{3}(\rho)=\iint \psi(x-y) h(y) R_{\rho}^{(2)}(d x, d y) .
$$

However a serious problem here is that we are operating on the right side of the inequality and cannot afford the luxury of Fatou's lemma. We have to have an actual identification of limit and therefore have to prove that the functionals

$$
H_{3}(\omega)=\sum \psi\left(x_{i}-x_{k}\right) h\left(N\left(x_{k}-y\right)\right)
$$

are uniformly integrable and can be truncated to yield uniformly bounded versions with uniformly small errors. What we can use for this is an estimate of the form

$$
E^{f_{N}} \int\left|\sum_{i, k} \psi\left(N\left(x_{i}-x_{k}\right)\right) h\left(N\left(x_{k}-y\right)\right)\right|^{4 / 3} d y \leqq C .
$$

$a$ and $b$ are uniformly bounded and cause no problems.

Lemma 7.9. If $f_{N} \in \mathscr{A}_{N, B, D}$, then

$$
E^{f_{N}} \int\left|\sum_{i, k} \psi\left(N\left(x_{i}-x_{k}\right)\right) \hat{H}\left(N\left(x_{k}-y\right)\right)\right|^{4 / 3} d y \leqq C,
$$

where $C$ depends only on $B$ and $D$. Proof. Let us denote $\psi\left(N\left(x_{\imath}-x_{k}\right)\right)$ by $\psi_{j k}, h\left(N\left(x_{k}-y\right)\right)$ by $h_{k}$ and $\sum_{i} \psi_{\imath k}$ by $T_{k}$.
Then

$$
\begin{aligned}
& \left|\sum_{i, k} \psi_{i k} h_{k}\right|=\left|\sum h_{k} T_{k}\right| \leqq\left(\sum h_{k}\right)^{1 / 2}\left(\sum h_{k} T_{k}^{2}\right)^{1 / 2} \\
& \left|\sum_{i k} \psi_{i k} h_{k}\right|^{4 / 3} \leqq\left(\sum h_{k}\right)^{2 / 3}\left(\sum h_{k} T_{k}^{2}\right)^{2 / 3} \\
& E^{f_{N}} \int\left|\sum_{i, k} \psi_{i k} h_{k}\right|^{4 / 3} d y \leqq E^{f_{N}}\left\{\int\left(\sum h_{k}\right)^{4 / 3}\left(\sum h_{k} T_{k}^{2}\right)^{2 / 3} d y\right\} \\
& \leqq\left(E^{f_{N}} \int\left(\sum h_{k}\right)^{2} d y\right)^{1 / 3}\left(E^{f_{N}} \int\left(\sum h_{k} T_{k}^{2}\right) d y\right)^{2 / 3} .
\end{aligned}
$$


But

$$
E^{f_{N}} \int\left|\sum h\left(N\left(x_{k}-y\right)\right)\right|^{2} d y=\frac{1}{N} E^{f_{N}} \sum \chi\left(N\left(x_{i}-x_{J}\right)\right)
$$

is bounded. Moreover

$$
E^{f_{N}} \int \sum h\left(N\left(x_{k}-y\right)\right) T_{k}^{2} d y=E^{f_{N}} \frac{1}{N} \sum_{i}\left|\sum_{j} \psi\left(N\left(x_{i}-x_{j}\right)\right)\right|^{2},
$$

and this was shown to us uniformly bounded before in Lemma 5.1.

Turning to our original estimate now we have

$$
\lim _{N \rightarrow \infty} E^{f_{N}} T_{4}=E^{Q}\left[\iint \lambda g(\lambda(x-y)) \rho(x) d x d y \int \hat{H}_{3}(\rho) \pi_{y}(d \rho)\right] .
$$

We now look at the final term

$$
\lim _{N \rightarrow \infty} E^{f_{N}} T_{6}=4 E^{Q}\left[\iint \chi(\lambda(x-y)) \rho(x) \rho(y) d x d y\right] .
$$

Combining (7.15) with (7.16) through (7.19) we obtain for any measure $Q$ in $\hat{\mathscr{A}}_{b, D}$,

$$
\begin{aligned}
E^{Q} & {\left[\int_{S} d x\left[\int \hat{H}_{\delta}(\rho) \pi_{x}(d \rho)\right]\right.} \\
& \left.\left.+\int_{S} d x\left[\int \hat{H}_{1, \delta}(\rho) \pi_{x}(d \rho)\right)\right]\right] \\
& \leqq E^{Q}\left[\iint \lambda g(\lambda(x-y)) \rho(x) \rho(y) d x d y\right. \\
& \left.+\iint \lambda g(\lambda(x-y)) \rho(x) d x d y \int \hat{H}_{3}(\rho) \pi_{y}(d \rho)\right] \\
& +\sqrt{D}\left[4 E^{Q}\left[\iint \chi(\lambda(x-y)) \rho(x) \rho(y) d x d y\right]\right]^{1 / 2} .
\end{aligned}
$$

In the relation (7.20) the right-hand side is clearly bounded uniformly in terms of our constants $B$ and $D$ for each fixed $\lambda$. We first let $\delta \rightarrow 0$ in the left. From the factoring properties of the correlation functions $R_{\rho}^{(2)}(d x, d y)$ and $R_{\rho}^{(3)}(d x, d y, d z)$ we can conclude by an application of Fatou's lemma

$$
\lim _{\delta \rightarrow 0} \int_{S} d z \int\left[\hat{H}_{\delta}(\rho)+\hat{H}_{1, \delta}(\rho)\right] \pi_{x}(d \rho) \geqq \int_{S} d x \int \rho P(\rho) \pi_{x}(d \rho),
$$

since by Theorem $10.4 P(\rho) \sim \rho^{2}$ for large $\rho$, it follows that

Lemma 7.10. For $Q \in \hat{\mathscr{A}}_{B, D}$,

$$
\sup _{Q \in \mathscr{A}_{B, \nu}} E^{Q}\left[\int \rho^{3}(x) d x\right] \leqq E^{Q}\left[\int_{S} d x \int \rho^{3} \pi_{x}(d \rho)\right]<\infty .
$$


We are interested in letting $\lambda \rightarrow \infty$ on the right-hand side. Let us look at each of the three terms on the right. Clearly $\iint \lambda g(\lambda(x-y)) \rho(x) \rho(y) d x d y$ $\leqq \int \rho^{2}(x) d x$, and since $E^{Q} \int \rho^{2}(x) d x<\infty$ we can use the dominated convergence theorem to conclude that

$$
\lim _{\lambda \rightarrow \infty} E^{Q} \iint \lambda g(\lambda(x-y)) \rho(x) \rho(y) d x d y=E^{Q} \int \rho^{2}(x) d x .
$$

The second term involves

and

$$
\iint \lambda g(\lambda(x-y)) \rho(x) d x d y \int \hat{H}_{3}(\rho) \pi_{y}(d \rho)
$$

$$
\lim _{\lambda \rightarrow \infty} \iint \lambda g(\lambda(x-y)) \rho(x) d y \int \hat{H}_{3}(\rho) \pi_{y}(d \rho)=\int \rho(y) \int \hat{H}_{3}(\rho) \pi_{y}(d \rho) .
$$

We can bound

$$
\begin{aligned}
& \left.\iint \lambda g(x-y)\right) \rho(x) d x d y \int P(\rho) \pi_{y}(d \rho) \\
& {\left[\int\left(\int \lambda g(\lambda(x-y)) \rho(x) d x\right)^{3}\right]^{1 / 3}\left[\int\left[\int P(\rho) \pi_{y}(d \rho)\right]^{3 / 2} d y\right]^{2 / 3}} \\
& \quad \leqq \frac{1}{3} \int \lambda g(\lambda(x-y)) \rho^{3}(x) d x d y+\frac{2}{3} \int[P(\rho)]^{3 / 2} \pi_{y}(d \rho) d y \\
& \quad \leqq A \text { by Lemma 7.10. }
\end{aligned}
$$

The third term clearly goes to zero as $\lambda \rightarrow \infty$. Therefore for every $Q \in \mathscr{A}_{B, D}$,

$$
E^{Q}\left[\int_{S} d x \int \rho P(\rho) \pi_{x}(d \rho)\right] \leqq E^{Q}\left[\int_{S} d x\left[\int \rho \pi_{x}(d \rho) \int P(\rho) \pi_{x}(d \rho)\right] .\right.
$$

Proof of Theorem 7.6. We can think of Eq. (7.22) as

$$
E^{Q} \int_{S} d x\left\{\int \rho P(\rho) \pi_{x}(d \rho)-\int \rho \pi_{x}(d \rho) \int P(\rho) \pi_{x}(d \rho)\right\} \leqq 0
$$

This means that on the average the covariance

$$
\int \rho P(\rho) \pi_{x}(d \rho)-\int \rho \pi_{x}(d \rho) \int P(\rho) \pi_{x}(d \rho)
$$

is nonnegative. However

$$
\begin{aligned}
& \int \rho P(\rho) \pi_{x}(d \rho)-\int \rho \pi_{x}(d \rho) \int P(\rho) \pi_{x}(d \rho) \\
& \quad=\int\left(\rho-\int \rho \pi_{x}(d \rho)\right)\left(P(\rho)-P\left(\int \rho \pi_{x} d \rho\right)\right) \pi_{x}(d \rho) \\
& \quad \geqq 0,
\end{aligned}
$$

because $P(\rho)$ is a nondecreasing function of $\rho$. The strictly increasing nature of $P(\rho)$ dictates that we have strict positivity unless $\pi_{x}(d \rho)$ is degenerate. This completes the proof of Theorem 7.6.

Having proved Theorem 7.6 the first application is an estimate on the regularity of densities in the support of any $Q \in \mathscr{A}_{B, D}$. 
Theorem 7.11. For any $Q \in \hat{\mathscr{A}}_{B, D}$,

$$
E^{Q}\left[\int_{S}\left\{[P(\rho(\theta))]_{\theta}\right\}^{2} \frac{1}{\rho(\theta)}\right] \leqq 2 D .
$$

Proof. Let us consider $k$ functions $u_{1}(x), \ldots, u_{k}(x)$ from $C^{\infty}(S)$ and $l$ functions $F_{1}(x), \ldots, F_{l}(x)$ also from $C^{\infty}(S)$. We consider as well the functions $g_{1}, \ldots, g_{k}$ from $C_{0}^{\infty}\left(R^{l}\right)$. Let us denote by $\hat{x}=\left(x_{1}, \ldots, x_{N}\right)$ a point of $S^{N}$. Then we define on $S^{N}$

$$
\begin{aligned}
& U_{n}(\hat{x})=\frac{1}{N} \sum_{j=1}^{N} u_{r}\left(x_{j}\right), \\
& G_{r}(\hat{x})=g_{r}\left(\frac{1}{N} \sum_{J=1}^{N} F_{1}\left(x_{j}\right), \ldots, \frac{1}{N} \sum_{J=1} F_{l}\left(x_{j}\right)\right) .
\end{aligned}
$$

We can perform integration by parts and write

$$
\begin{aligned}
& \int\left\{\sum_{r=1}^{k}\left[L_{N} U_{r}(\hat{x})\right]\left[G_{r}(\hat{x})\right]\right\} f_{N}(\hat{x}) d \mu_{N} \\
& =-\frac{1}{2} \int\left\{\sum_{r=1}^{k}\left\langle\nabla U_{r}, \nabla G_{r}\right\rangle+\sum_{r=1}^{k}\left\langle U_{r}, \frac{\nabla f_{n}}{f_{N}}\right\rangle G_{r}\right\} f_{N} d \mu_{N} \\
& =T_{1}+T_{2} .
\end{aligned}
$$

Since $\left|\frac{\partial G_{r}}{\partial x_{J}}\right| \leqq \frac{C}{N}$ it follows by an elementary calculation that $\left|T_{1}\right| \rightarrow 0$ as $N \rightarrow \infty$.

We can estimate $T_{2}$ by Schwartz's inequality to obtain

$$
\left|T_{2}\right| \leqq \frac{1}{2} \sqrt{2 D}\left(\int \frac{1}{N} \sum_{j=1}^{N}\left(\sum_{r=1}^{k} u_{r}^{\prime}\left(x_{j}\right) G_{r}(\hat{x})\right)^{2} \cdot f_{N} d \mu_{N}\right)^{1 / 2}
$$

The left-hand side of (7.24) can be calculated to be approximately

$$
\frac{1}{2} \int \sum_{r=1}^{k}\left\{\frac{1}{N} \sum_{j=1}^{N} u_{r}^{\prime \prime}\left(x_{j}\right) G_{r}(\hat{x})\left(1+\sum_{i=1}^{N} \psi\left(N\left(x_{i}-x_{j}\right)\right)\right)\right\} f_{N} d \mu_{N}
$$

For any $Q \in \hat{\mathscr{A}}_{B, D}$, we can therefore obtain the inequality

$$
\begin{aligned}
& \frac{1}{2}\left|E^{Q} \int \sum_{r=1}^{k} u_{r}^{\prime \prime}(x) G_{r}\left(\left\langle\rho, F_{1}\right\rangle, \ldots,\left\langle\rho, F_{l}\right\rangle\right) P(\rho(x)) d x\right| \\
& \quad \leqq \frac{1}{2} \sqrt{2 D}\left[E^{Q} \int\left|\sum u_{r}^{\prime}(x) G_{r}\left(\left\langle\rho, F_{1}\right\rangle, \ldots,\left\langle\rho, F_{l}\right\rangle\right)\right|^{2} \phi(x) d x\right]^{1 / 2} .
\end{aligned}
$$

It is not difficult to pass from this estimate to

$$
\left|E^{Q} \int \psi_{x x}(x, \omega) P(\rho(x, \omega)) d x\right| \leqq \sqrt{2 D}\left\{E^{Q} \int \psi_{x}^{2}(x, \omega) \rho(x, \omega) d x\right\} .
$$

Here $\omega$ is the generic point and $Q$ is the measure on random densities $\rho(\cdot)$.

The estimate (7.25) will hold if the test function $\psi(x, \omega)$ is uniformly bounded along with $\psi_{x}$ and $\psi_{x x}$. Since we can replace $\psi_{x}$ by $\psi$ we can assume 


$$
\left|E^{Q} \int \psi_{x}(x, \omega) P(\rho(x, \omega)) d x\right| \leqq \sqrt{2 D}\left\{E^{Q} \int \psi^{2}(x, \omega) \rho(x, \omega) d x\right\}^{1 / 2}
$$

for all bounded $\psi$ with $\psi_{x}$ bounded and having $\int \psi d x \equiv 0$ for almost all $\omega$.

Since $E^{Q} \int \rho^{2}(x, \omega) d x$ has a uniform bound in $\hat{\mathscr{A}}_{B, D}$ we will have a bound of the form

$$
\left|E^{Q} \int \psi_{x}(x, \omega) P(\rho(x, \omega)) d x\right| \leqq C\left(E^{Q} \int \psi^{4}(x, \omega) d x\right)^{1 / 4},
$$

provided $\int \psi(x, \omega) d x \equiv 0$. This is enough to yield the estimate

$$
E^{Q} \int\left|[P(\rho(x, \omega))]_{x}\right|^{4 / 3} d x \leqq C .
$$

We can now rewrite $(7.26)$ in the form

$$
\left|E^{Q} \int \psi(x, \omega)(P(\rho(x, \omega)))_{x} d x\right| \leqq \sqrt{2 D}\left\{E^{Q} \int \psi^{2}(x, \omega) \rho(x, \omega) d x\right\}^{1 / 2}
$$

for all $\psi(x, \omega)$ such that $E^{Q} \int \psi^{4}(x, \omega) d x<\infty$ and satisfy $\int \psi(x, \omega) d x \equiv 0$. Let $\psi(x, \omega)$ be any function with $E^{Q} \int \psi^{4}(x, \omega) d x<\infty$. We write $\psi(x, \omega)=\psi_{1}(x, \omega)+h(\omega)$, where $\psi_{1}(x, \omega)$ has the property $\int \psi_{1}(x, \omega) d x \equiv 0$. Then because $(P(\rho(x, \omega)))_{x}$ is orthogonal to constants,

$$
\begin{aligned}
&\left|E^{Q} \int \psi(x, \omega) P(\rho(x, \omega))_{x} d x\right| \\
&=\left|E^{Q} \int \psi_{1}(x, \omega)(P(\rho(x, \omega)))_{x} d x\right| \\
& \leqq \sqrt{2 D}\left\{E^{Q} \int[\psi(x, \omega)-h(\omega)]^{2} P(x, \omega) d x\right\}^{1 / 2} \\
& \leqq \sqrt{2 D}\left\{E^{Q} \int[\psi(x, \omega)-h(\omega)]^{2}\right. \\
&\cdot[1+\rho(x, \omega)] d x\}^{1 / 2} .
\end{aligned}
$$

Since $h(\omega)=0 \int \psi(x, \omega) d x$ we can bound

Therefore

$$
E^{Q} h^{2}(\omega) \leqq E^{Q} \int \psi^{2}(x, \omega)[1+\rho(x, \omega)] d x .
$$

$$
\left|E^{Q} \int \psi(x, \omega)(P(\rho(x, \omega)))_{x} d x\right| \leqq C\left[E^{Q} \int \psi^{2}(x, \omega)[1+\rho(x, \omega)] d x\right]^{1 / 2}
$$

for all functions $\psi(x, \omega)$ with $E^{Q} \int \psi^{4}(x, \omega) d x<\infty$. This is enough to conclude that

$$
E^{Q} \int \frac{\left\{P(\rho(x, \omega))_{x}\right\}^{2}}{1+\rho(x, \omega)} d x<\infty
$$



We now go back to (7.29) and use for $\psi(x, \omega)$ the function $\frac{[P(\rho(x, \omega))]_{x}}{\varepsilon+\rho(x, \omega)}$. (This
has to be justified.) Then $h(\omega)=0$.

We now obtain

$$
\begin{aligned}
& E^{Q} \int \frac{\left[P(\rho(x, \omega))_{x}\right]^{2}}{\varepsilon+\rho(x, \omega)} d x \\
& \quad \leqq \sqrt{2 D}\left(E^{Q} \int \frac{(P(\rho(x, \omega)))_{x}^{2}}{[\varepsilon+\rho(x, \omega)]^{2}} \rho(x, \omega) d x\right)^{1 / 2} \\
& \quad \leqq \sqrt{2 D}\left(E^{Q} \int \frac{(P(\rho(x, \omega)))_{x}^{2}}{\varepsilon+\rho(x, \omega)} d x\right)^{1 / 2}
\end{aligned}
$$

Because of (7.30) we can let $\varepsilon \rightarrow 0$ and obtain our theorem.

Now we have all the ingredients necessary to prove Theorem 7.3. If we calculate

$$
\begin{aligned}
& \lim _{\lambda \rightarrow 0} \limsup _{\varepsilon \rightarrow 0} \limsup _{N \rightarrow \infty} \sup _{Q \in \mathscr{A}_{N, B, D}} E^{Q}\left[\int\left|\rho_{\lambda}(x)-\rho_{N \varepsilon}(x)\right| d x\right] \\
& \quad=\lim _{\lambda \rightarrow 0} \sup _{Q \in \mathscr{\mathscr { A }}_{B, D}} E^{Q}\left[\int\left|\int h_{\lambda}(x-y) \rho(y) d y-\rho(x)\right| d x\right] \\
& \quad=0
\end{aligned}
$$

because of the estimate in Theorem 7.11. Because $P(\rho)$ is strictly monotone and continuous in $\rho$ any control on $\int \frac{(P(\rho))_{\theta}^{2}}{\rho} d \theta$ will provide control on the $L_{1}$
modulus of continuity of $\rho(\cdot)$.

\section{Hydrodynamic Limit}

Let $Q_{N}$ be the family of probability measures induced on $C\left[[0, T] ; M_{1}(S)\right]$ by looking at the trajectory $\frac{\delta_{x_{1}(\cdot)}+\cdots \delta_{x_{n}(\cdot)}}{\varepsilon}$ in the space of probability measures. We have already established Theorem 3.1 asserting the compactness of $Q_{N}$. Let $Q$ be any weak limit point. The results of Sect. 7 establish Theorems 3.2 and 3.3. All we need is the following lemma.

Lemma 8.1. If we have the bounds

$$
\begin{aligned}
& E^{Q} \int \rho^{2}(\theta, \omega) d \theta \leqq C, \\
& E^{Q} \int \frac{(P(\rho))_{\theta}^{2}}{\rho} d \theta \leqq C,
\end{aligned}
$$

then for some other constant $C$,

$$
E^{Q} \int \rho^{3}(\theta) d \theta \leqq C
$$


Proof. Let us consider

$$
\begin{aligned}
S(\rho) & =[1+P(\rho)]^{3 / 4} \\
(S(\rho))_{\theta} & =\frac{3}{4}[1+P(\rho)]^{-1 / 4}[P(\rho)]_{\theta}, \\
\int(S(\rho))_{\theta}^{2} d \theta & \leqq \frac{9}{16} \int \frac{(P(\rho))_{\theta}^{2}}{[1+P(\rho)]^{1 / 2}} d \theta \\
& \leqq C
\end{aligned}
$$

because of the bound $P(\rho) \geqq c \rho^{2}$ for large $\rho$. Since $P(\rho) \geqq C \rho^{2}$ we have $S(\rho) \geqq$ $C \rho^{3 / 2}$ for large $\rho$ and we have $S(\rho) \leqq C \rho^{3 / 2}$ with another constant. In other words we can control $\int S(\rho) d \theta$ and $\int(S(\rho))_{\theta}^{2} d \theta$, and hence control $\int S^{2}(\rho) d \theta$.

Remark. Although the conclusion of the lemma is already implied by Lemma 7.10 , it follows from the first two estimates assumed in the lemma.

\section{Uniqueness}

Let $\rho_{0}(\theta)$ be a function on $L_{2}(S)$. Assume $\rho(t, \theta)$ is a weak solution of

$$
\frac{\partial \rho(t, \theta)}{\partial t}=\frac{1}{2}(P(\rho(t, \theta)))_{\theta \theta}
$$

with

$$
\rho(0, \theta)=\rho_{0}(\theta) .
$$

Assume that the solution $\rho(t, \theta)$ has the property

$$
\int_{S} \int_{0}^{T} \rho^{3}(t, \theta) d \theta d t<\infty
$$

and

$$
\int_{S} \int_{0}^{T} \frac{[P(\rho(t, \theta))]_{\theta}^{2}}{\rho(t, \theta)} d t d \theta<\infty .
$$

Theorem 9.1. A weak solution satisfying (9.1) and (9.2) is unique.

Let if possible $u(t, \theta)$ and $v(t, \theta)$ be two solutions corresponding to the same initial data $\rho_{0}(\theta)$. Let us define the function

$$
\omega(\theta, t)=\frac{1}{2} \int_{0}^{t}\left\{[P(u(s, \theta))]_{\theta}-[P(v(s, \theta))]_{\theta}\right\} d s .
$$

Using the fact that $\iint_{0}^{t} \rho(s, \theta) d s d \theta<\infty$, it follows from (9.2) that

$$
[P(u)]_{\theta}-[P(v)]_{\theta} \in L_{1}(S \times[0, t]) .
$$

Hence $\omega(\theta, t)$ is well defined as a continuous function of $t$ for almost all $\theta$. An elementary computation yields

$$
\frac{\partial \omega}{\partial \theta}=u-v
$$


in the sense of distributions. Now

$$
\begin{aligned}
\frac{\partial}{\partial t}[\omega(\theta, t)]^{2} & =2 \omega(\theta, t) \frac{\partial w}{\partial t} \\
& =\omega(\theta, t)[P(u)-P(v)]_{\theta} .
\end{aligned}
$$

We want to show that

$$
\iint_{0}^{t}|\omega(\theta, t)|\left\{\left|[P(u)]_{\theta}\right|+\mid[P(v)]_{\theta}\right\} d \theta d t<\infty .
$$

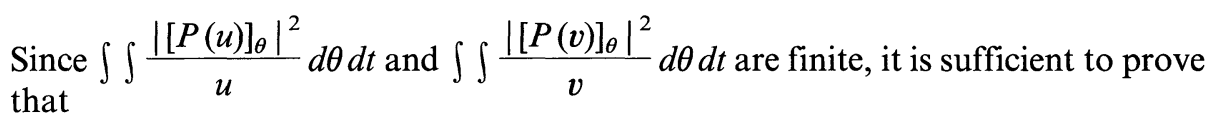

and

$$
\iint_{0}^{t}|\omega(\theta, t)|^{2} u(\theta, t) d \theta d t
$$

$$
\iint_{0}^{t}|\omega(\theta, t)|^{2} v(\theta, t) d \theta d t
$$

are finite. (9.1) and (9.4) are sufficient now to conclude (9.5). We therefore conclude that

$$
\chi(\theta, t)=[\omega(\theta, t)]^{2}
$$

is a continuously differentiable map of $[0, t]$ into $L_{1}(S)$. Moreover $\omega(\theta, t) \rightarrow 0$ a.e. $\theta$ as $t \rightarrow 0$,

$$
\begin{aligned}
\int \frac{\partial \chi}{\partial t} d \theta & =\int \omega(\theta, t)[P(u(\theta, t))-P(v(\theta, t))]_{\theta} d \theta \\
& =-\int\left(\frac{\partial \omega}{\partial \theta}\right)(P(u)-P(v)) d \theta \\
& =-\int(u-v)(P(u)-P(v)) d \theta \leqq 0
\end{aligned}
$$

Therefore $\int \chi(\theta, t) d \theta \equiv 0$. This proves uniqueness.

\section{Appendix}

In this section we will establish some results concerning finite and infinite volume Gibbs measures for our continuous system with finite range interaction. Although these results are not new and are part of the folklore in the field, there does not appear to be any source that we can refer to for a precise statement of the results in the form we need them. We will therefore provide a proof.

Our main assumption is that we have a repulsive pair potential, i.e. a function $V(x)$, which is nonnegative, even, continuously differentiable and has compact support. We denote by $\left[-c_{0}, c_{0}\right]$ the interval of support of the function $V(\cdot)$ and we assume further that $\psi(x)=-x V^{\prime}(x) \geqq 0$ and that $V(0)>0$.

For any $l>0$, we consider the exterior $[-l, l]^{c}$ of the interval $[-l, l]$ relative to the real line. A configuration $\omega$ is the realization of a point process and 
represents a locally finite collection of points. Since our interaction has range $c_{0}$ we are only interested in the part of the realization that has to do with the border $\left[-l-c_{0},-l\right] \cup\left[l, l+c_{0}\right]$ and therefore we can think of $\omega$ as an arbitrary but finite configuration of points in the border.

We shall think of $\omega=\left\{y_{a}\right\}$, as a finite set of points located in $\left[-l-c_{0},-l\right] \cup\left[l, l+c_{0}\right]$. The canonical partition function $Z(n, l, \omega)$ is defined as follows for $n \geqq 0, l>0$ and all $\omega$,

$$
\begin{gathered}
Z(n, l, \omega)=1 \text { if } n=0 \text { for all } l \text { and } \omega, \\
Z(n, l, \omega)=\frac{1}{n !} \int_{-l}^{l} \ldots \int_{-l}^{l} \exp \left[-\sum_{i \neq j=1}^{n} V\left(x_{i}-x_{j}\right)\right. \\
\left.-2 \sum_{i, \alpha} V\left(x_{i}-y_{\alpha}\right)\right] d x_{1} \ldots d x_{n} .
\end{gathered}
$$

The canonical Gibbs measure $\mu_{n, l, \omega}$ is a point process on $[-l, l]$ with exactly $n$ points or equivalently a measure on $[-l, l]^{n}$ with a density given by

$$
f_{n, l, \omega}\left(x_{1}, \ldots, x_{n}\right)=c_{n} \exp \left[-\sum_{i \neq j=1}^{n} V\left(x_{i}-x_{j}\right)-2 \sum_{i, \alpha} V\left(x_{i}-y_{\alpha}\right)\right] \text {. }
$$

The normalizing constant $c_{n}$ is of course given by

$$
c_{n}^{-1}=n ! Z(n, l, \omega) \text {. }
$$

When $\omega$ is the empty set we denote $Z(n, l, \omega)$ by $Z_{n, l}^{0}$ and $\mu_{n, l, \omega}$ by $\mu_{n, l}^{0}$.

The grand canonical partition function with activity $\lambda$ is defined for every real $\lambda$ and

$$
\hat{Z}(\lambda, l, \omega)=\sum_{n=0}^{\infty} e^{\lambda n} Z(n, l, \omega),
$$

and the grand canonical Gibbs measure is a point process $\hat{\mu}_{\lambda, l, \omega}$ on $[-l, l]$ which is thought of as a probability measure on

It is given by

$$
\bigcup_{n=0}^{\infty}[-l, l]^{n}
$$

$$
\hat{\mu}_{\lambda, l, \omega}=[\hat{Z}(\lambda, l, \omega)]^{-1} \sum e^{\lambda n} Z(n, l, \omega) \mu_{n, l, \omega},
$$

i.e. it is a convex combination of canonical Gibbs measures and the weights are proportional to $e^{\lambda n} Z(n, l, \omega)$.

Although we have only defined these Gibbs measures on intervals of the form $[-l, l]$ it is clear that they enjoy translation invariance of sorts and can be defined on any interval $[a, b]$ and depend only on the configuration in the appropriate border. Moreover, there is an internal consistency in the following two senses.

First, by definition, the canonical Gibbs measure is the conditional distribution of the grand canonical Gibbs measure when it is conditioned by the number of particles in the set.

Moreover if we take the grand canonical Gibbs distribution on $[-l, l]$ and condition it with respect to the configuration in $[a, b]^{c}$, where $-l \leqq a<b \leqq l$, then 
the conditional distribution is the grand canonical Gibbs measure on $[a, b]$ with the same activity $\lambda$.

The infinite volume Gibbs measure $\mu_{\lambda}$ corresponding to activity $\lambda$, is defined as a stationary point process such that for every interval $[a, b]$ the conditional distribution of the configuration in $[a, b]$ given the configuration in $[a, b]^{c}$ is the grand canonical Gibbs measure $\hat{\mu}_{\lambda, l, \omega}$ with $l=\frac{1}{2}(b-a)$ and $\omega$ representing the exterior configuration. We shall state the main theorems we will use in our article and provide a sketch of the proof at the end, after establishing a few key estimates as lemmas.

Theorem 10.1. The following thermodynamic functions exists:

$$
\begin{aligned}
& F(\lambda)=\lim _{l \rightarrow \infty} \frac{1}{2 l} \log \hat{Z}_{\lambda, l}^{0} \text { for } \lambda \text { real }, \\
& \Psi(\rho)=\lim _{\substack{l \rightarrow \infty \\
n \rightarrow \infty \\
n / 2 k \rightarrow \rho}} \frac{1}{2 l} \log Z_{n, k}^{0} \text { for } \rho \geqq 0 .
\end{aligned}
$$

The function $F(\lambda)$ is convex in $\lambda$ and the function $\Psi(\rho)$ is concave in $\rho$. Moreover they are related by the equation

$$
F(\lambda)=\sup _{\rho}[\lambda \rho+\Psi(\rho)]
$$

Theorem 10.2. For each value of the activity parameter $\lambda$, there exists exactly one Gibbs measure $\mu_{\lambda}$ with activity $\lambda$. The point process $\mu_{\lambda}$ depends continuously on the parameter $\lambda$. As a point process its density is given by

$$
\rho(\lambda)=F^{\prime}(\lambda)=\frac{d F(\lambda)}{d \lambda} .
$$

In particular $F(\lambda)$ is once continuously differentiable. The function $\rho(\lambda)$ is continuous and nondecreasing. Its inverse is

We have in addition

$$
\lambda=-\Psi^{\prime}(\rho)=\frac{d \Psi(\rho)}{d \rho} .
$$

$$
\lim _{\lambda \rightarrow \infty} \rho(\lambda)=\infty, \quad \lim _{\lambda \rightarrow-\infty} \rho(\lambda)=0 .
$$

If $I$ is a unit interval and $N(I)$ denotes the number of points in the interval $I$, then

$$
E^{\mu_{\lambda}}\left[\{N(I)\}^{r}\right]<\infty \quad \text { for every } r \geqq 1 .
$$

Theorem 10.3. Let $n \rightarrow \infty$ and $l \rightarrow \infty$ in such a manner that $\frac{n}{l} \rightarrow \rho$. Let $H(\omega)$ be a bounded continuous local function depending on the configuration in some fixed finite interval $\left[-l_{0}, l_{0}\right]$. Let us denote by $H_{x}(\omega)$ the same functional evaluated at the configuration in $\left[-l_{0}+x, l_{0}+x\right]$. We denote by $\hat{H}(\lambda)$ the expected value $E^{\mu \lambda}[H(\omega)]$. Then for every $\varepsilon>0$,

$$
\lim \mu_{n, l}^{0}\left[\left|\frac{1}{2 l} \int_{-\left(l-l_{0}\right)}^{\left(l-l_{0}\right)} H_{x}(\omega) d x-\hat{H}(\lambda)\right| \geqq \varepsilon\right]=0,
$$

where $\rho=F^{\prime}(\lambda)$. 
Moreover for every function $H(x)$ on $[-1,1]$ which is nonnegative, has compact support with $\int \phi(x) d x=1$,

$$
\lim \mu_{n, l}^{0}\left[\left|\frac{1}{l} \sum \phi\left(\frac{x_{i}}{l}\right)-\rho\right| \geqq \varepsilon\right]=0 .
$$

Remark 1. It follows from (10.5) that if $\lambda_{1}, \lambda_{2}$ are two different activities such that $F^{\prime}\left(\lambda_{1}\right)=F^{\prime}\left(\lambda_{2}\right)=\rho$, then $\hat{H}\left(\lambda_{1}\right)=\hat{H}\left(\lambda_{2}\right)$ for all functions $H(\omega)$. This in turn implies $\mu_{1}=\mu_{\lambda_{1}}$ and $\lambda_{1}=\lambda_{2}$. Therefore $\rho(\lambda)$ is a strictly increasing function of $\lambda$ and its inverse $-\Psi^{\prime}(\rho)$ is a strictly increasing continuous function of $\rho$.

We define the function

$$
P(\rho)=F(\lambda(\rho)) .
$$

Theorem 10.4. There exists constants $0<c_{1}<c_{2}<\infty$ such that

$$
c_{1} \rho^{2} \leqq P(\rho) \leqq c_{2} \rho^{2} \text { for } \rho \text { large . }
$$

Since $E^{\mu_{\lambda}}\left[\{N(I)\}^{r}\right\}<\infty$ for every $\mu_{\lambda}$, we consider for every function $f\left(x_{1}, \ldots, x_{k}\right)$ of $k$-variables which is smooth and has compact support on $R^{k}$, the variable

$$
\xi_{f}=\sum f\left(x_{1}, \ldots, x_{k}\right),
$$

where the summation is over all $k$-tuples of $k$ distinct points in the configuration. $\xi_{f}$ has a finite expectation and

$$
E^{\mu_{\lambda}} \xi_{f}=\int f\left(x_{1}, \ldots, x_{k}\right) R_{k}^{\lambda}\left(d x_{1}, \ldots, d x_{k}\right) .
$$

The measure $R_{k}^{\lambda}\left(d x_{1}, \ldots, d x_{k}\right)$ is the $k$-point correlation measure. It has translation invariance relative to $x_{1}, \ldots, x_{k} \rightarrow x_{1}+a, \ldots, x_{k}+a$. The one point measure $R_{k}^{\lambda}\left(d x_{1}\right)=\rho(\lambda) d x_{1}$, where $\rho(\lambda)$ is the density.

We need the following identity between $P(\rho)$ and $R_{2}^{\lambda}\left(d x_{1}, d x_{2}\right)$.

Theorem 10.5. If $\lambda$ and $\rho$ are related by $\lambda=\lambda(\rho)$, then

$$
P(\rho)=\rho+\int \psi\left(x_{1}-x_{2}\right) \phi\left(x_{2}\right) R_{1}^{\lambda}\left(d x_{1}, d x_{2}\right),
$$

where $\phi(x)$ is any function on $R$ that is smooth, has compact support, is nonnegative and has $\int \phi(x) d x=1$.

Finally the ergodicity of $\mu^{\lambda}$ implies some asymptotic factorization of the correlation measures.

Theorem 10.6. Let $g_{1}, g_{2}$ be functions on $R$ that are smooth and have compact support. Let $g_{3}$ be a smooth function on $R^{2}$ having compact support. Then for any $\lambda$,

$$
\begin{gathered}
\lim _{k \rightarrow 0} k \int g_{1}\left(k\left(x_{1}-x_{2}\right)\right) g_{2}\left(x_{2}\right) R_{2}^{\lambda}\left(d x_{1}, d x_{2}\right)=\rho^{2}\left(\int g_{1}(x) d x\right)\left(\int g_{2}(x) d x\right), \\
\lim _{k \rightarrow 0} k \int g\left(k\left(x_{1}-x_{2}\right)\right) g_{2}\left(x_{2}, x_{3}\right) R_{3}^{\lambda}\left(d x_{1}, d x_{2}, d x_{3}\right) \\
=\rho\left(\int g_{1}(x) d x\right)\left(\int g_{2}\left(x_{2}, x_{3}\right) R_{2}^{\lambda}\left(d x_{1}, d x_{2}\right)\right) .
\end{gathered}
$$

We adopt the following conventions regarding constants. Constants will always be independent of $n, l$ and $\omega . A, B, C$ will denote constants and will be 
used at different times of denote different constants. We will use $\gamma$ to denote constants that are strictly positive. The constant $c_{0}$ is the range of the interaction $V(\cdot)$. The quantity $\frac{n}{2 l}$ will be denoted by $\rho$.

Lemma 10.7. For every $l>c_{0}$ and $\omega$ we have

$$
Z_{n, l-c_{0}}^{0} \leqq Z(n, l, \omega) \leqq Z_{n, l}^{0} .
$$

Proof. For $n=0$, there is nothing to prove. The upper bound is obtained by dropping the interaction terms involving the points in the border. For the lower bound we limit the integration to the situation where all the points $x_{1}, \ldots, x_{n}$ lie inside $\left[-\left(l-c_{0}\right),\left(l-c_{0}\right)\right]$.

Lemma 10.8. There is a constant $C$ such that

$$
\frac{d \log Z_{n, l}^{0}}{d l} \leqq \frac{n}{l}+C \frac{n^{2}}{l^{2}}
$$

Proof. By differentiation we can write

$$
\frac{d \log Z_{n, l}^{0}}{d l}=\frac{n}{l}+\frac{1}{l} \int \sum \psi\left(x_{i}-x_{j}\right) d \mu_{n, l}^{0} .
$$

[Change variables $x_{i}=l y_{i}$, differentiate and change back.] From formula (4.4) we have an estimate

$$
\sum \psi\left(x_{i}-x_{j}\right) \leqq C_{1} \sum V\left(x_{i}-x_{j}\right)
$$

for some $C_{1}$. Since

$$
d \mu_{n, l}^{0}=c_{n} \exp \left[-\sum V\left(x_{\imath}-x_{j}\right)\right] d \theta,
$$

where $d \theta$ is volume on $[-l, l]^{N}$, it follows that

$$
\int \sum V\left(x_{i}-x_{j}\right) d \mu_{n, l}^{0} \leqq \int \sum V\left(x_{i}-x_{j}\right) d \hat{\theta},
$$

where $d \hat{\theta}$ is normalized volume. We can now calculate $\int \sum V\left(x_{i}-x_{j}\right) d \hat{\theta}$ and obtain (10.12).

Lemma 10.9. For every $n$ and $l>0$,

$$
z_{n+1, l}^{0} \leqq \frac{2 l}{n+1} Z_{n, l}^{0}
$$

Proof. If we ignore any interaction involving $x_{n+1}$ and integrate out $x_{n+1}$ we obtain (10.13).

Lemma 10.10. Let $l=l_{1}+l_{2}+\frac{1}{2} c_{0}$ with $l_{1}>0, l_{2}>0$. Then

$$
Z_{n, l}^{0} \geqq \sum_{\substack{n_{1}+n_{2}=n \\ n_{1} \geqq 0, n_{2} \geqq 0}} Z_{n, l_{1}}^{0} Z_{n_{2}, l_{2}}^{0} .
$$

In particular for any $n_{1}, n_{2}$ with $n_{1}+n_{2}=n$,

$$
Z_{n, l}^{0} \geqq Z_{n_{1}, l_{1}}^{0} Z_{n_{2}, l_{2}}^{0} \text {. }
$$


Proof. Let us divide the interval $[-l, l]$ into three parts $I_{1}, I$ and $I_{2}$ with lengths $2 l_{1}, c_{0}$ and $2 l_{2}$ arranged in order. We limit the integration to the domain where $I_{1}$ and $I_{2}$ contain all the points $\left(x_{1}, \ldots, x_{n}\right)$. Here $n_{1}$ and $n_{2}$ represent the number of particles in $I_{1}$ and $I_{2}$ respectively. The combinatorial factors adjust themselves and we get Lemma 10.10 .

Lemma 10.11. There exist constants $A$ and $B$ such that

$$
\mu_{n, l, \omega} \leqq A e^{B \rho^{2}} \mu_{n, l}^{0}, \quad \text { provided } l \geqq 2 c_{0} .
$$

Proof. The density $f_{n, l, \omega}$ would be dominated by $f_{n, l}^{0}$ if it were not for the normalizing constant. We therefore need only estimate the ratio

$$
Z_{n, l}^{0}[Z(n, l, \omega)]^{-1} \text {. }
$$

Lemmas 10.7 and 10.8 will establish a bound for this if we can keep $l$ away from $c_{0}$.

Lemma 10.12. There are positive constants $\gamma, c_{1}$ and $c_{2}$ such that

$$
\frac{(2 l)^{n}}{n !} \exp \left[-c_{2} \frac{n^{2}}{l}\right] \leqq Z_{n, l}^{0} \leqq \frac{(2 l)^{n}}{n !} \exp \left[-\gamma \frac{n^{2}}{l}+c_{1} n\right] \text {. }
$$

Proof.

$$
\frac{n ! Z_{n, l}^{0}}{(2 l)^{n}}=\frac{1}{(2 l)^{n}} \int_{-l}^{l} \ldots \int_{-l}^{l} \exp \left[-\sum_{i \neq J=1}^{n} V\left(x_{l}-x_{J}\right)\right] d x_{1} \ldots d x_{n}
$$

The lower bound follows from Jensen's inequality. On the other hand there are constants $\gamma>0$ and $c_{1}$ such that

$$
\sum_{i \neq j=1}^{n} V\left(x_{l}-x_{j}\right) \geqq \gamma \frac{n^{2}}{l}-c_{1} n
$$

for every configuration $\left(x_{1}, \ldots, x_{n}\right)$.

Lemma 10.13. Let $I \subset[-l, l]$ be an arbitrary interval of length 1 . Let $E_{k}$ denote the event that the set I contains exactly $k$ of the points from $\left(x_{1}, \ldots, x_{n}\right)$. Then there are constants $A, B, C$ and $v>0$ such that

$$
\mu_{n, l}^{0}\left(E_{k}\right) \leqq A \exp \left[-v k^{2}+B k \rho+c \rho^{2}\right]
$$

for all $n$ and $l>\frac{1}{2}$.

Proof. Let $i=[a, a+1]$ for some $-l \leqq a \leqq l-1$. Let $I_{1}=[-l, a]$ and $I_{2}=[a+1, l]$. We let $l_{1}=\frac{l+a}{2}$ and $l_{2}=\frac{1}{2}(l-a-1)$. Suppose that $I_{1}, I_{2}$ contain $n_{1}, n_{2}$ points respectively and $n_{1}+n_{2}+k=n$. If we ignore all interactions between points in different intervals and use the bound

we obtàin

$$
\sum_{\substack{x_{i}, x_{j} \in I \\ i \neq j}} V\left(x_{i}-x_{j}\right) \geqq v_{1} k^{2}-c_{1} k
$$

$$
\mu_{n, l}^{0}\left(E_{k}\right) \leqq \frac{1}{k !} \exp \left[-v_{1} k^{2}+c_{1} k\right] \cdot \frac{1}{Z_{n, l}^{0}} \sum_{n_{1}+n_{2}=n-k} Z_{n_{1}, l_{1}}^{0} Z_{n_{2}, l_{2}}^{0} .
$$


From Lemma 10.10,

$$
\begin{aligned}
\sum_{n_{1}+n_{2}=n-k} Z_{n_{1}, l_{1}}^{0} Z_{n_{2}, l_{2}}^{0} & \leqq Z_{n-k, l_{1}+l_{2}+c_{0} / 2}^{0} \\
& =Z_{n-k, l-(1 / 2)+\left(c_{0} / 2\right)}^{0} .
\end{aligned}
$$

We can now apply (10.14) to get

$$
Z_{n, l+c_{0}+(k l / n)}^{0} \geqq Z_{n-k, l-(1 / 2)+\left(c_{0} / 2\right)}^{0} \cdot Z_{k,(1 / 2)+(k l / n)}^{0} \cdot
$$

(10.17) then becomes

$$
\mu_{n, l}^{0}\left(E_{k}\right) \leqq \frac{1}{k !} \exp \left[-v_{1} k^{2}+c_{1} k\right] \frac{Z_{n, l+c_{0}+(k l / n)}^{0}}{Z_{n, l}^{0}} \cdot \frac{1}{Z_{k,(1 / 2)+(k l / n)}^{0}} .
$$

We can use bounds from Lemma 10.8 and the lower bound of Lemma 10.12 to complete the proof.

Lemma 10.14. If $N_{I}$ is the number of particles in the interval I then for some $v_{1}>0$ and $l>\frac{1}{2}$,

$$
E^{\mu_{n, l}^{0}}\left[\exp \left\{v_{1} N_{I}^{2}\right\}\right] \leqq A \exp \left[B \rho^{2}\right]
$$

where $A$ and $B$ are some constants.

Proof. We pick $v_{1}<v$ and sum

$$
\sum \mu_{n, l}^{0}\left(E_{k}\right) e^{v_{1} k^{2}}
$$

using the bounds in Lemma 10.13.

Lemma 10.15. For every $m$, there exists a constant $c_{m}$ such that

$$
E^{\mu_{n, l}^{0}}\left[N_{I}^{m}\right] \leqq C_{m}(1+\rho)^{m}
$$

for all $n$ and $l$.

Proof. For any random variable $X$ there is a constant $c_{m}$ independent of $X$ such that

$$
E|X|^{m} \leqq c_{m}\left(1+\log E e^{X^{2}}\right)^{m / 2}
$$

If we now combine Lemma 10.11 with Lemmas 10.14 and 10.15 we get

Lemma 10.16. For all $l>\frac{1}{2}$ and $n$,

$$
\begin{gathered}
\sup _{\omega} \mathrm{E}^{\mu_{n, l}^{\omega}}\left[\exp \left[v N_{I}^{2}\right]\right] \leqq A \exp \left[B \rho^{2}\right], \\
\sup _{\omega} E^{\mu^{\omega_{n, l}}}\left[\left[N_{I}\right]^{m}\right] \leqq C_{m}(1+\rho)^{m} .
\end{gathered}
$$
them.

We now turn to the grand canonical Gibbs distribution and estimates on

Lemma 10.17. Let $\hat{\mu}_{\lambda, l, \omega}$ be the grand canonical Gibbs measure. Then for any given $\lambda_{0}>0$ for all $\lambda \leqq \lambda_{0}, l>\frac{1}{2}$ and $\omega$ we have the following bounds:

$$
\begin{gathered}
E^{\hat{\mu}_{\lambda, l, \omega}}\left[\exp \left\{v N_{I}^{2}\right\}\right] \leqq A \exp \left[B\left(1+\lambda_{0}\right)^{2}\right], \\
E^{\hat{\mu}_{\lambda, l, \omega}}\left[\left[N_{I}\right]^{m}\right] \leqq C_{m}\left(1+\lambda_{0}\right)^{m} .
\end{gathered}
$$


Proof. According to Lemma 10.17 there exists $\alpha>0$ such that

$$
E^{\mu^{\omega_{n, l}}}\left[\exp \left[\alpha N_{I}^{2}\right]\right] \leqq A \exp \left[B \rho^{2}\right] .
$$

We can replace $\alpha$ by $\varepsilon \alpha$ and then $B$ can be replaced by $\varepsilon \beta$ by Hölder's inequality.

We pick $\varepsilon$ so small that $\varepsilon B$ is less than $\frac{v}{2}$, where $v$ is as in Lemma 10.13 . We will continue to denote $\varepsilon \alpha$ by $\alpha$. We now have

$$
E^{\mu_{n, l, \omega}}\left[\exp \left[\alpha N_{I}^{2}\right]\right] \leqq A \exp \left[\frac{v}{2} \rho^{2}\right]
$$

To estimate $E^{\hat{\mu}_{\lambda, l, \omega}}\left[\exp \left[N_{I}^{2}\right]\right.$ we must bound

$$
\begin{aligned}
& \frac{A}{\hat{Z}(\lambda, l, \omega)} \sum e^{\lambda n} Z(n, l, \omega) \exp \left[\frac{v}{2} \rho^{2}\right]=T, \\
& \mathrm{~T}^{2 l} \leqq \frac{a^{2 l}}{\hat{Z}(\lambda, l, \omega)} \sum e^{\lambda n} Z(n, l, \omega) \exp \left[\frac{v}{2}(2 l) \rho^{2}\right] .
\end{aligned}
$$

We use the trivial bound $\hat{Z}(\lambda, l, \omega) \geqq 1$. Then

$$
T^{2 l} \leqq A^{2 l} \sum_{n=0}^{\infty} e^{\lambda n} \frac{(2 l)^{n}}{n !} e^{-v\left(n^{2} / l\right)+c n+(r / 4) \cdot\left(n^{2} / l\right)} .
$$

One can estimate $\frac{(2 l)^{n}}{n !}$ by $e^{2 l}$ and obtain $(10.21)$ by direct estimation of the sum (more or less Gaussian sum). The inequality (10.22) follows from (10.21). The fact that the summation is over nonnegative $n$ makes the estimates uniform for $\lambda$ in $\left(-\infty, \lambda_{0}\right]$.

Now we turn our attention to a proof of our theorems.

Proof of Theorem 10.1. The existence of the free energy $F(\lambda)$ and the specific energy or entropy functional $\Psi(\rho)$ follows from standard subadditivity arguments. Lemma 10.10 provides the required subadditivity as well as the concavity. The formula for $\hat{Z}$ in terms of $Z$ can be used with a standard Laplace asymptotic formula to calculate the free energy in terms of the function $\Psi(\rho)$ i.e. to prove formula (10.4).

Proof of Theorem 10.2. For any real $\lambda$, we consider the family $\hat{\mu}_{\lambda, l}^{0}$ of grand canonical Gibbs measures as point processes on $[-l, l]$. We let $l \rightarrow \infty$. Lemma 10.17 allows us to take a subsequence and prove the existence of a Gibbs measure which may perhaps be nonstationary. A standard averaging argument over translations and another limit produces a stationary candidate.

For uniqueness let $P_{1}, P_{2}$ be two grand canonical Gibbs measures for the same activity $\lambda$. Let $[-a, a]$ be any arbitrary finite interval and we denote by $\left\|P_{1}-P_{2}\right\|_{a}$ the variation norm on the $\sigma$ field configurations on $[-a, a]$.

We have to show that $\left\|P_{1}-P_{2}\right\|_{a}=0$ for every $a$. Let us take $J_{k}=\left[x:(k-1) c_{0} \leqq|x| \leqq k c_{0}\right]$ and define the conditional expectation operator,

$$
\left(T_{k} F\right)(\omega)=\int F\left(\omega^{\prime}\right) \hat{\mu}_{\lambda, k c 0, \omega}\left(d \omega^{\prime}\right),
$$

taking functions on the configuration space of $J_{k}$ into those of $J_{k+1}$. Proving 
uniqueness can easily be reduced to proving

$$
\left\|T_{k} F|\|\leqq \theta\|| F \mid\right\|
$$

for some $\theta<1$ for all $k$ and $F$. Here

$$
\begin{aligned}
\|F \mid\| & =\frac{1}{2} \sup _{\omega_{1}, \omega_{2}}\left|F\left(\omega_{1}\right)-F\left(\omega_{2}\right)\right| \\
& =\inf _{c} \sup _{\omega}|F(\omega)-c|,
\end{aligned}
$$

where the infimum is taken over constants. This however needs only the following lemmas.

Lemma 10.18. Let $E_{k}$ be the event that the interval $J_{k}$ contains no points of the configuration. Then there is $a \delta>0$ depending only on $\lambda$ such that

$$
\hat{\mu}_{\lambda, k c_{0}, \omega}\left(E_{k}\right) \geqq \delta
$$

for all $k \geqq 1$ and $\omega$.

Proof. All we need is to estimate the ratio

$$
\begin{aligned}
\tau & =\frac{1}{\hat{Z}\left(\lambda, k c_{0}, \omega\right)}\left(\sum_{n=0}^{\infty} e^{\lambda n} Z\left(n, k c_{0}, \omega\right) \mu_{n, k c_{0}, \omega}\left(E_{k}\right)\right) \\
\tau \geqq & \frac{1}{\hat{Z}\left(\lambda, k c_{0}, \omega\right)} \sum_{n=0}^{\infty} e^{\lambda n} Z_{n,(k-1) c_{0}}^{0} \\
& \geqq \frac{\sum_{n=0}^{\infty} e^{\lambda n} Z_{n,(k-1) c_{0}}^{0}}{\sum_{n=0}^{\infty} e_{\lambda n} Z_{n, k c_{0}}^{0}} .
\end{aligned}
$$

We use the estimate

$$
\frac{Z_{n,(k-1) c_{0}}^{0}}{Z_{n, k c_{0}}} \geqq e^{-A \rho^{2}-B}
$$

from Lemma 10.8 and we write

$$
\begin{aligned}
\tau & \geqq \frac{\sum_{n=0}^{\infty} e^{\lambda n} Z_{n, k c_{0}}^{0} e^{-A(n / 2 l)^{2}}}{\sum_{n=0}^{\infty} e^{\lambda n} Z_{n, k c_{0}}^{0}} \cdot e^{-B} \\
& \geqq e^{-B} e^{-A R^{2}}\left(1-\sum_{n \geqq 2 l R} e^{\lambda n} Z_{n, k c_{0}}^{0} e^{-A\left(n / 2 l^{2}\right.}\right) .
\end{aligned}
$$

We have used $\sum_{n=0}^{\infty} e^{\lambda n} Z_{n, k c 0}^{0} \geqq 1$.

Now we use the estimates of Lemma 10.12 to complete the proof. 
Continuous dependence is an easy consequence of uniform bounds and uniqueness.

Uniqueness of the Gibbs measure yields the differentiability of $F(\lambda)$ and the continuity of $\rho(\lambda)$. The bounds on the moments of $N(I)$ are contained in Lemma 10.17. The relation between $F^{\prime}(\lambda)$ and $\Psi^{\prime}(\rho)$ is a consequence of the Legendre transform (10.4). To see the limits of $\rho(\lambda)$ as $\lambda \rightarrow \pm \infty$, the behavior as $\lambda \rightarrow-\infty$ will follow from the easily established relation $\lim _{\lambda \rightarrow-\infty} F(\lambda)=0$. The behavior for $\lambda \rightarrow \infty$ is obtained by proving $c_{1} \lambda^{2} \leqq F(\lambda) \leqq c_{2} \lambda^{2}$ as $\lambda \rightarrow \infty$. These are deduced from Lemma 10.12 .

Proof of Theorem 10.3. This theorem, known often as the theorem on the equivalence of ensembles, is an easy consequence of large deviation theory. We note first that the uniqueness of Gibbs measures implies that each $\mu_{\lambda}$ is ergodic, and in fact, for nice local functions $H(\omega)$,

$$
\varlimsup_{l \rightarrow \infty} \frac{1}{2 l} \log \mu_{\lambda}\left\{\left|\frac{1}{2 l} \int_{-l}^{l} H_{x}(\omega) d x-\hat{H}(\lambda)\right| \geqq \varepsilon\right\} \leqq-\eta(\varepsilon)<0 .
$$

If $E_{l}$ is the event that the set $[-l-1,-l] \cup[l, l+1]$ has no points in it, then from Lemma 10.18 it follows easily that

$$
\mu_{\lambda}\left[E_{l}\right] \geqq \delta>0 \quad \text { uniformly as } \quad l \rightarrow \infty .
$$

Combining (10.23) and (10.24)

$$
\varlimsup_{l \rightarrow \infty} \frac{1}{2 l} \log \hat{\mu}_{\lambda, l}^{0}\left\{\left|\frac{1}{2 l} \int_{-l}^{l} H_{x}(\omega) d x-y Z H(\lambda)\right| \geqq \varepsilon\right\} \leqq-\eta(\varepsilon)<0 .
$$

One has a similar result for the number of points $N(l)$ in the interval $[-l, l]$,

$$
\varlimsup_{l \rightarrow \infty} \frac{1}{2 l} \log \mu_{\lambda, l}^{0}\left\{\left|\frac{N(l)}{2 l}-\rho(\lambda)\right| \geqq \varepsilon\right\} \leqq-\eta(\varepsilon)<0 .
$$

In particular

$$
\varlimsup_{l \rightarrow \infty} \mu_{\lambda, l}^{0}\left\{\left|\frac{N(l)}{2 l}-\rho(\lambda)\right| \leqq \varepsilon\right\}=1
$$

If we look at the ratios

$$
\frac{\mu_{\lambda, l}^{0}[N(l)=k+1]}{\mu_{\lambda, l}^{0}[N(l)=k]}=\frac{Z_{k+1, l}^{0}}{Z_{k, l}^{0}}
$$

in the range where $\left|\frac{k}{2 l}-\rho(\lambda)\right| \leqq \varepsilon$, then Lemma 10.9 provides an upper bound for the ratio and Lemmas 10.10 and 10.8 provide a lower bound for it. Since $\rho(\lambda)>0$ we can make the bounds uniform over $k$ in the range we are interested in. In turn this means

$$
\mu_{\lambda, l}^{0}\left[\left|\frac{N(l)}{2 l}-\rho(\lambda)\right| \leqq \varepsilon\right] \leqq e^{c l \varepsilon} \cdot 2 l \varepsilon \cdot \inf _{|(k / 2 l)-\rho(\lambda)| \leqq \varepsilon} \mu_{\lambda, l}^{0}[N(l)=k] .
$$


It follows now from (10.28) that

$$
\lim _{\substack{l \rightarrow \infty \\ k \rightarrow \infty \\ k / 2 l \rightarrow \rho(\lambda)}} \frac{1}{2 l} \log \mu_{\lambda, l}^{0}[N(l)=k]=0 .
$$

If we combine (10.23) with (10.29) we obtain the first half of Theorem 10.3. The second half is similar. We start with the estimate from large deviations.

$$
\varlimsup_{l \rightarrow \infty} \frac{1}{2 l} \log \hat{\mu}_{\lambda}\left\{\left|\sum \phi\left(l x_{l}\right)-\rho(\lambda)\right| \geqq \varepsilon\right\} \leqq-\eta(\varepsilon)<0
$$

if $\phi$ is nonnegative, is supported on $[-1,1]$, and has $\int \phi(x) d x=1$. This covers Theorem 10.3 except when $n / 2 l \rightarrow 0$. But this case is trivial anyway since most intervals in $[-l, l]$ would have to be empty.

Proof of Theorem 10.4. It follows from the bounds in Lemma 10.2 that for $\lambda \rightarrow+\infty$ there exist constants $0<c_{1}<c_{2}<\infty$ such that

$$
c_{1} \lambda^{2} \leqq F(\lambda) \leqq c_{2} \lambda^{2}
$$

Since $F(\lambda)$ is convex, this implies for some $0<c_{1}<c_{2}<\infty$,

$$
c_{1} \lambda \leqq \rho(\lambda)=F^{\prime}(\lambda) \leqq c_{2} \lambda \text {. }
$$

Now because $P(\rho)=F(\lambda(\rho))$, (10.31) and (10.32) imply our theorem.

Proof of Theorem 10.5. We have the identity from Lemma 10.8,

$$
\begin{aligned}
\frac{d \log Z_{m, l}^{0}}{d l}= & \frac{n}{l}+\frac{1}{l} \int \sum \psi\left(x_{i}-x_{j}\right) d \mu_{n l}^{0} \\
= & \frac{n}{l}+\frac{1}{l} \iint_{-l}^{l} \sum \psi\left(x_{i}-x_{j}\right) \phi\left(x_{j}-x\right) d x d \mu_{n l}^{0} \\
& + \text { a small error } .
\end{aligned}
$$

Clearly

$$
\lim _{l \rightarrow \infty} \frac{d Z_{n, l}^{0}}{d l}=2 \rho+2 \int \Psi\left(x_{1}-x_{2}\right) \phi\left(x_{2}\right) R_{2}^{\lambda}\left(d x_{1}, d x_{2}\right)
$$

Since $Z_{n, l}^{0} \sim 2 l \Psi(\rho)=2 l \Psi\left(\frac{n}{2 l}\right)$,

$$
\frac{d Z_{n, l}^{0}}{d l} \simeq 2 \Psi\left(\frac{n}{2 l}\right)-2 \cdot l \cdot \frac{n}{2 l^{2}} \Psi^{\prime}\left(\frac{n}{2 l}\right)
$$

Therefore

$$
\begin{aligned}
\lim _{l \rightarrow \infty} \frac{d Z_{n, l}^{0}}{d l} & =2 \Psi(\rho)-2 \rho \Psi^{\prime}(\rho) \\
& =2 \Psi(\rho)+2 \lambda \rho \\
& =2 F(\lambda) .
\end{aligned}
$$


Combining (10.33) and (10.34)

$$
P(\rho)=F(\lambda)=\rho+\int \psi\left(x_{1}-x_{2}\right) \phi\left(x_{2}\right) R_{2}^{\lambda}\left(d x_{1}, d x_{2}\right) .
$$

Proof of Theorem 10.6. Consider

$$
\xi_{k, f}=\sum f_{k}\left(x_{1}, x_{2}\right)
$$

where $f_{k}\left(x_{1}, x_{2}\right)=k g_{1}\left(k\left(x_{1}-x_{2}\right)\right) g_{2}\left(x_{2}\right)$,

$$
\lim _{k \rightarrow \infty} \xi_{k, f}=\rho \sum g_{2}\left(x_{2}\right) \text { a.e. by the ergodic theorem . }
$$

If we take expectations with respect to $\mu_{\lambda}$ we obtain (10.9). Relation (10.10) is similar. Lemma 10.17 provides enough uniform integrability to justify interchanging limits and integration.

\section{References}

1. Guo, M.Z., Papanicolaou, G.C.: Self diffusion of interacting Brownian particles, Probabilistic Methods in Mathematical Physics, pp. 113-151. Ito, K, Ikeda N. (eds.). Tokyo: Kinokuniya 1987

2. Guo, M.Z., Papanicolaou, G.C., Varadhan, S.R.S.: Nonlinear diffusion limit for a system with nearest neighbor interaction. Commun. Math. Phys. 118, 31-59 (1988)

3. Spohn, H.: Equilibrium fluctuations for interacting Brownian particles. Commun. Math. Phys. 103, 1-33 (1986)

4. Olla, S., Varadhan, S.R.S.: Scaling limit for interacting Ornstein-Uhlenbeck Processes. Commun. Math. Phys. 135, 355-378 (1991)

5. Ruelle, David: Statistical mechanics; rigorous results. New York: Benjamin 1969

Communicated by J.L. Lebowitz 
